\title{
Washboard modes as ELM-related events in JET
}

\author{
C P Perez ${ }^{1,2}$, H R Koslowski ${ }^{1}$, T C Hender ${ }^{3}$, P Smeulders ${ }^{4}$, A Loarte ${ }^{5}$,

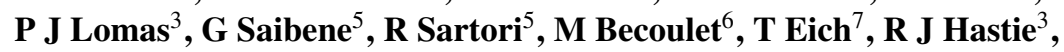 \\ G T A Huysmans ${ }^{6}$, S Jachmich ${ }^{1}$, A Rogister ${ }^{1}$, F C Schüller ${ }^{2}$ and \\ JET EFDA contributors ${ }^{8}$
}

1 Association EURATOM-Forschungszentrum Jülich, Institut für Plasmaphysik, Trilateral Euregio Cluster, D-52425 Jülich, Germany

${ }_{2}^{2}$ FOM-Rijnhuizen, Ass. EURATOM-FOM, TEC, PO Box 1207, 3430 BE Nieuwegein, NL

${ }^{3}$ EURATOM/UKAEA Fusion Association, Culham Science Centre, Abingdon OX14 3DB, UK

${ }^{4}$ Associazione EURATOM-ENEA sulla Fusione, Centro Ricerche Frascati, C.P. 65, 00044-Frascati (Rome), Italy

${ }^{5}$ EFDA-CSU, Max-Planck-Institut für Plasmaphysik, Garching D-85748, Germany

${ }^{6}$ Association EURATOM-CEA, Cadarache, F-13108 St Paul-lez-Durance, France

${ }^{7}$ Max-Planck-Institut für Plasmaphysik, EURATOM Assoziation, Garching D-85748, Germany

E-mail: c.perez.von.thun@fz-juelich.de

Received 21 March 2003

Published 26 November 2003

Online at stacks.iop.org/PPCF/46/61 (DOI: 10.1088/0741-3335/46/1/005)

\begin{abstract}
Washboard (WB) modes (Smeulders P et al 1999 Plasma Phys. Control. Fusion 41 1303) are a very common edge instability regularly observed in the H-mode regime in JET. They are detected as (normally several) bands of continuously fluctuating magnetic activity rotating in the direction of the electron diamagnetic drift with typical frequencies in the range of $10-90 \mathrm{kHz}$. The time evolution of the WB mode frequency is found to follow qualitatively the evolution of the electron temperature measured near the pedestal top, probably due to the strong diamagnetic drift associated with the large pedestal gradients. Evidence for their involvement in the pedestal and ELM dynamics will be presented. Increasing WB mode amplitude is correlated with an increase in the time between consecutive type-I ELMs. In situations in which a sudden increase (decrease) of WB mode activity is observed, the build-up of the pedestal temperature (and, linked to this, also of the pedestal pressure) of the electrons is seen to become slower (faster). This is a strong indication that the WB mode activity has a regulating effect on the pedestal and that it is responsible for an enhanced transport of energy across the separatrix. The occurrence of a class of type-I ELM precursor modes commonly observed in JET in discharges with low to moderate collisionality $\left(v_{\mathrm{e}}^{*}<2\right.$, roughly) (Perez C P et al EFDA-JET Preprint EFD-P(02)11) is found to be associated with a weakening of the WB modes. The underlying mechanism for this interaction has not
\end{abstract}

8 See annexe of J Pamela et al 2003 Overview of JET results Proc. 19th Int. Conf. on Fusion Energy 2002 (Lyon, 2002) (Vienna: IAEA). 
been yet identified. In contrast to low triangularity discharges, WB activity is found to increase with gas puffing at high triangularity. This can provide an explanation for the regime recently identified on JET that has been called the mixed type-I/type-II ELM regime (Saibene G et al 2002 Plasma Phys. Control. Fusion 44 1769). A modified version of the peeling-ballooning cycle for type-I ELMs on JET that takes into account the WB mode phenomenon and is consistent with the experimental observations is proposed.

This article was due to be published in issue 9 of Plasma Phys. Control. Fusion. To access this special issue, please follow this link: http://www.iop.org/ EJ/toc/0741-3335/45/9.

\section{Introduction}

The energy losses associated with type-I ELMs [1-3] are a serious concern for ITER due to the possibly unacceptable transient heat loads expected on the divertor tiles $[4,5]$. Our understanding of type-I ELMs, in particular their origin and the underlying processes governing their dynamics, is still incomplete. From the MHD point of view, research is increasingly focusing on the role of the most promising ideal instabilities, namely (finite- $n$ ) ballooning and kink-(peeling) modes, and more recently also on coupled ballooning-kink modes, leading to the proposition of a so-called peeling-ballooning cycle for type-I ELMs [6].

Washboard (WB) modes are a very robust phenomenon commonly observed in JET $\mathrm{H}$-mode discharges and are not thought to belong to one of the above-mentioned instabilities. Since their discovery [7], rather little attention has been paid to them, either theoretically or experimentally. So far they have not been regarded as an ELM-relevant instability. However, in this paper evidence for their involvement in the pedestal and ELM dynamics will be presented.

This paper is organized as follows. In section 2 an overview of WB properties is given, recalling earlier findings from [7] where appropriate. Section 3 is devoted to the observation of an exclusive interaction between the WB modes and a class of type-I ELM precursor modes, commonly observed at JET in discharges with low to moderate collisionality. The relationship between the WB modes and a regime recently identified on JET that has been called the mixed type-I/type-II ELM regime is discussed in section 4. Building on these findings, a modified version of the peeling-ballooning cycle for type-I ELMs that takes into account the physics of WB modes will be proposed in section 5. Finally, a summary and a discussion of the results are given.

\section{WB properties}

Figure 1 shows a spectrum of toroidal mode numbers for an example ELMy H-mode, where the colours denote the toroidal mode numbers $n$. The $n$-numbers are obtained by making a time-windowed Fourier decomposition of the signals of a toroidal set of Mirnov coils and analysing the relative phase shift of the fluctuations. Depending on the data acquisition, the signals have either a $250 \mathrm{kHz}$ or $1 \mathrm{MHz}$ sampling rate. For the analysis a high resolution array of five coils, with toroidal angles, $\Delta \phi$, gradually increasing from $1.7^{\circ}$ to $15.9^{\circ}$, has been used, adequate for mode numbers $n \leqslant 11$. A subset of the coils with lower $\Delta \phi$ was employed if higher mode numbers needed to be resolved or simply to check the correctness of previous calculations. To reduce the noise level of the plots, points were discarded when the amplitudes were below a user-defined threshold or the fitting error of the mode number exceeded a certain amount. Toroidal mode number spectra are convenient for discriminating the various types of 


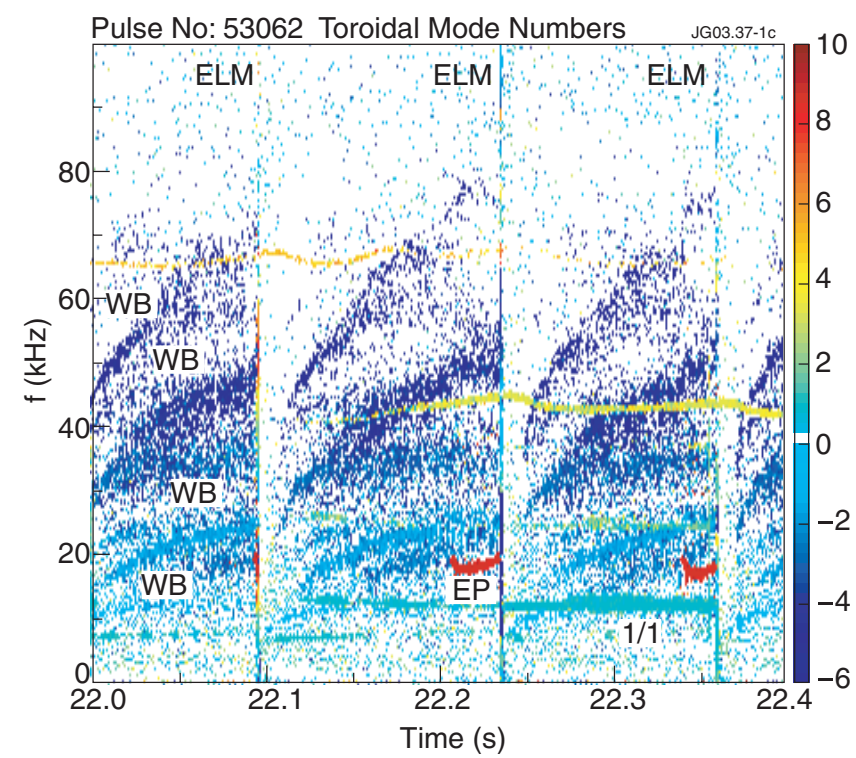

Figure 1. Spectrum of toroidal mode numbers, $n$. Three type-I ELMs occur at 22.096, 22.24 and 22.36 s. The red-coloured modes $(n=+8)$ are type-I ELM precursors [8]. The WB modes are the multiple blue-coloured broader bands propagating in the direction of the electron diamagnetic drift (negative $n$-numbers).

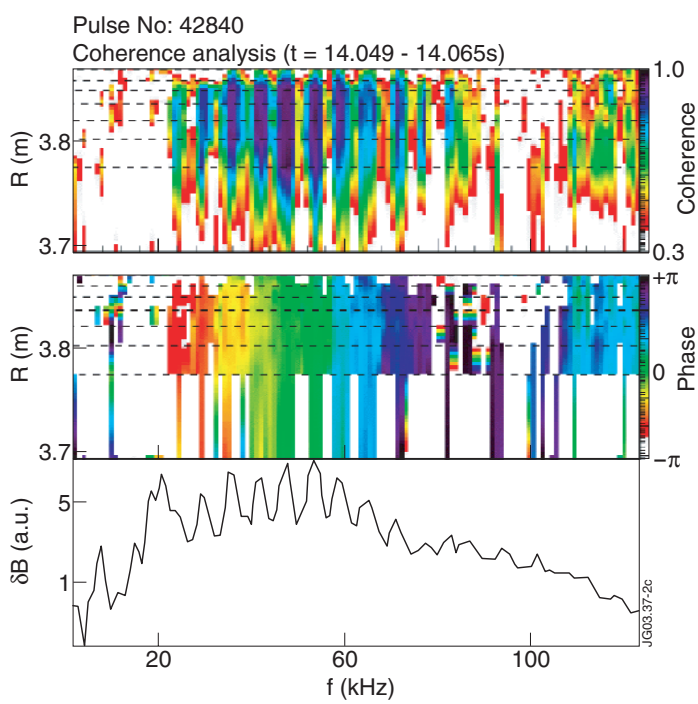

Figure 2. Coherence and phase spectra as a function of frequency and major radius, $R$, for a set of multiple WB bands (taken from [7]). The separatrix as calculated by EFIT is at $R=3.86 \mathrm{~m}$. The colour scales are shown at the right of each plot. The dashed lines denote the measuring radii of the reflectometer channels. The WB bands occur at $\sim 20-75 \mathrm{kHz}$. The third plot shows the power spectrum of the reference Mirnov signal, where the individual WB bands are clearly visible.

MHD activity present. Due to its twofold filtering (in frequency- and in phase-space), they are very useful for finding activity that is often only faintly visible on magnetic spectrograms (like the WB modes), and will be used often throughout this paper. Positive and negative mode numbers are detected. Modes with negative $n$ rotate in the opposite direction (toroidally or 
poloidally) to modes with positive $n$. The convention used here is that modes with negative $n$ rotate in the direction of the electron diamagnetic drift. Three type-I ELMs occur at 22.096, 22.24 and $22.36 \mathrm{~s}$. In between them one can observe several (blue coloured) bands of activity with frequencies ranging from $\sim 10$ to $80 \mathrm{kHz}$ propagating in the direction of the electron diamagnetic drift. These are the WB modes. They typically have mode numbers in the range $n=-1$ to -8 .

In between ELMs, WB modes have a quite characteristic frequency evolution. Normally, both the WB frequency and the spectral extent are initially low when reappearing after an ELM and tend to increase gradually until interrupted by the next ELM. In longer ELM-free phases, in particular, the WB frequency reaches a steady value. In time-frequency plots the WB bands then sometimes resemble the periodic ridges of an old-fashioned washboard (from which their name originates). Generally, the WB frequency evolution is affected by the level of gas fuelling (as will be discussed later). In the example of figure 1 no fuelling is applied.

In figure 1 , the red-coloured modes $(n=+8)$ around $20 \mathrm{kHz}$ are the so-called type-I ELM precursor modes [8]. Both ELM precursor modes and WB modes are observed at pedestal radii. In contrast to the WB modes, the ELM precursors are observed to propagate in the direction of the ion diamagnetic drift. This indicates that WB modes belong to a different class of instabilities from the ELM precursor modes. In general, the range of ELM precursor $n$-numbers that have been observed is 1-13. Precursors with low $n \sim 1-3$ are known to be external kinks (also known at JET as outer modes $[9,10]$ ), while experimental findings and their comparison with stability calculations suggest that the precursor modes with higher $n$ are not pure external kinks but coupled kink-ballooning modes. The precursor modes are regularly detected in discharges with low to moderate (normalized) electron pedestal collisionality (up to $v_{\mathrm{e}}^{*} \sim 2$, roughly). At higher collisionality the modes seem to be absent, indicating that a sufficiently high edge current is important to destabilize the precursors. Long-lived precursors are often seen to grow and shrink repeatedly during their lifetime and can change their dominant $n$-numbers rapidly. Detailed studies of the type-I ELM precursors have been presented elsewhere [8]. We shall return to them later.

Analysis of the poloidal mode numbers, $m$, on the basis of poloidally distributed coils is more complicated. Due to the plasma toroidicity the apparent $m$-number depends on the poloidal angle where the coils are located (so-called $\theta^{*}$-effect $[11,12]$ ). Plasma shaping (elongation, triangularity) and the presence of an X-point impose further difficulties for modes located close to the plasma boundary. The X-point, in particular, can distort the poloidal mode structure considerably. Poloidal mode numbers determined by coils picking up magnetic fluctuations around the plasma midplane $(\theta=0, \pi)$ will then have the tendency to be lower than the real $m$-number of the mode. Only a limited number of coils, distributed poloidally around the plasma cross section, are available on JET. For the analysis, four coils are used, two on the low-field side (LFS) ( $6^{\circ}$ apart) and two on the high-field side (HFS) $\left(50^{\circ}\right.$ apart). Due to aliasing effects the set of coils is not suitable for analysis of modes with high $m$-numbers, but for lower $m(\lesssim 6)$ it has proved reasonably reliable for modes such as NTMs [13]. The procedure used for the analysis is described in more detail in appendix B of [7]. For the WB bands with the lowest $n(n=-1)$ the poloidal mode number analysis gives reproducible results, as summarized in table 1 . For each discharge two or three fast acquisition time windows have been analysed. Within each discharge the resulting $m$-numbers were not found to change significantly. The $m$-numbers obtained are comparable with, or are slightly larger than, $q_{95}$ and thus correspond to rational surfaces located close to the plasma boundary.

Figure 2 shows previous results of a coherence analysis for a hot ion $\mathrm{H}$-mode discharge [14] with particularly distinct WB modes (see also figure 8). The coherence analysis uses a reference Mirnov signal and channels of the JET edge reflectometer $[15,16]$ with cut-off densities ranging 
Table 1. Poloidal mode numbers, $m$, for WB bands with $n=-1$, obtained through the analysis of the phase shifts of the fluctuations measured by four poloidally distributed Mirnov coils. Also given for each discharge is the safety factor at $95 \%$ of the poloidal flux, $q_{95}$, calculated by EFIT.

\begin{tabular}{llll}
\hline Pulse no & $n$-Number & $m$-Number & $q_{95}$ \\
\hline 42840 & -1 & -4.0 to -4.5 & 3.8 \\
50846 & -1 & -3.0 to -3.5 & 3.3 \\
52308 & -1 & -3.2 to -3.7 & 3.2 \\
53062 & -1 & -4.0 to -5.0 & 3.6 \\
53299 & -1 & -3.2 to -3.7 & 3.2 \\
\hline
\end{tabular}

from 0.43 to $4.13 \times 10^{19} \mathrm{~m}^{-3}$. The upper plots give the values of the coherence and phase of the cross-spectral density, respectively, as a function of major radius, $R$ and frequency, $f$. The dashed lines denote the measuring radii of the reflectometer channels estimated on the basis of a density profile obtained with the core LIDAR [17]. In general, the resolution of the core LIDAR is about $12 \mathrm{~cm}$ and not sufficient to resolve properly the pedestal, but tends to overestimate its width. Therefore, the cut-off radii of the reflectometer channels are probably even closer to the separatrix, which EFIT predicts to be at $R=3.86 \mathrm{~m}$ (with an uncertainty of $\sim 1 \mathrm{~cm}$ ) for this case. It is difficult to give on this basis an accurate estimate of the radial extent of WB modes with respect to the width of the transport barrier, but it is certain that the WB modes are located at the plasma edge, occurring at least at pedestal radii but possibly extending further inwards into the plasma [7]. One can see further from figure 2 that WB modes show kink-like radial displacements with no radial phase inversions ('twisting parity'); hence they are not magnetic islands.

The amplitude of WB modes is not constant but appears to be continuously fluctuating. This can be generally observed in spectrograms or $n$-number spectra like figure 1 , where the individual WB bands are seen to be composed of intermittent pulsations rather than a continuous mode. The timescale of these fluctuations could not be accurately determined, but it seems to be comparable with the time resolution of the Fourier analysis. The amplitude fluctuations are also likely to be the cause of the relative broadness of the individual WB bands in terms of frequency. From the spectral width of the WB bands, the WB amplitude is estimated to fluctuate with a typical repetition rate of $1-3 \mathrm{kHz}$. The possibility that the frequency spread of each WB band originates from a set of WB modes with the same $n$ but located at slightly different edge radii is not supported. Changes in the WB mode phase across the reflectometer channels should then be observed in figure 2, which is not the case.

The displacements associated with the WB modes are small. For individual WB bands density displacements in the sub-millimetre range have been obtained previously from analysis of edge reflectometer data [7]. ECE measurements are of limited use for the study of WB modes because the noise of the ECE channels turns out to be at least of the same order as the WB-induced fluctuations and because only measurements from the inner part of the pedestal are available (further outwards towards the separatrix the plasma loses its optical thickness). Assuming that the real noise of the ECE channels is given by the thermal noise, which is determined by the pre- and postdetection bandwidth, for the optically thick radii an upper bound for the WB-induced displacements of roughly $1 \mathrm{~mm}$ is obtained, which is compatible with the reflectometry results. The small magnitude of the displacements is in contrast to the observed relative broad radial extent of the WB modes, which according to the data seems to be at least of the order of several centimetres.

Since WB modes rotate opposite to the core toroidal plasma rotation, the often observed gradual increase of WB frequency between ELMs cannot be explained in terms of toroidal 
plasma acceleration. This would require a reversal of the toroidal rotation velocity, $v_{\phi}$, at the transport barrier, with the reversed $v_{\phi}$ having to reach values in excess of $200 \mathrm{~km} \mathrm{~s}^{-1}$ to reproduce the measured WB frequencies. This implies an edge rotation that is comparable with the core rotation, but in the opposite direction, for which there is no evidence.

Experimentally, it has been repeatedly observed that a close relationship exists between the evolution of the WB frequency and the evolution of the edge electron temperature measured by ECE channels resonant close to the pedestal top $\left(T_{\mathrm{e}}^{\mathrm{ped}}\right)$. For the discharges analysed here an array of 48 ECE heterodyne radiometer channels was available, viewing the plasma horizontally from the LFS, slightly below the plasma midplane. The channels have a spatial resolution of typically $\sim 1-2 \mathrm{~cm}$. ECE data with a frequency response of $1 \mathrm{kHz}$ are available throughout the discharge, while for selected time windows fast ECE data with $250 \mathrm{kHz}$ sampling rate is available as well. Figure 3 gives a comparative plot for the frequency evolution of WB bands and the evolution of $T_{\mathrm{e}}^{\text {ped }}$, showing a good qualitative agreement in the evolution of both quantities. This suggests that the observed WB frequency behaviour originates from the

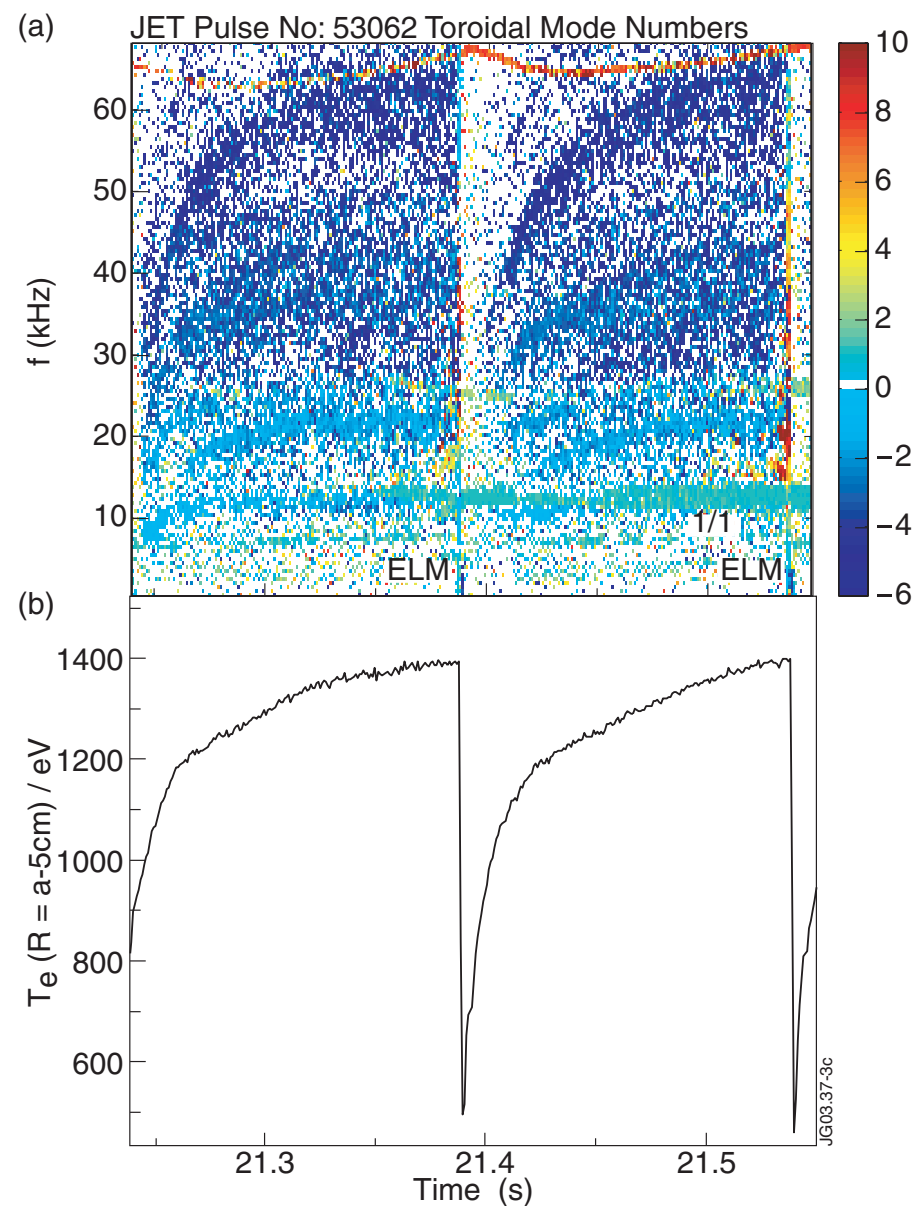

Figure 3. (a) Spectrum of toroidal mode numbers showing the WB frequency evolution and (b) electron temperature at the pedestal shoulder measured by means of ECE showing a close relationship between both quantities. In (a) the green-coloured mode (labelled 1/1) around $13 \mathrm{kHz}$ is a sawtooth precursor. 
increasing diamagnetic drift associated with the gradual pedestal recovery between ELMs. A further example is shown in figure 10, which shows that, unlike the edge temperature, the edge density does not affect the WB frequency noticeably. The following relation holds for the electron diamagnetic frequency:

$$
f_{* \mathrm{e}}(r, t) \sim \frac{p_{\mathrm{e}}^{\prime}}{n_{\mathrm{e}}}=\frac{n_{\mathrm{e}}^{\prime}}{n_{\mathrm{e}}} T_{\mathrm{e}}+T_{\mathrm{e}}^{\prime} .
$$

Due to diagnostic limitations, neither the gradients of the edge density and edge temperature nor their evolution in time are determined to a sufficient accuracy to allow a quantitative comparison of the edge diamagnetic drift with the WB mode frequency evolution. Since the density related contribution appears simultaneously on the numerator and the denominator of the first term of (1), and since for given radii both $n_{\mathrm{e}}$ and $n_{\mathrm{e}}^{\prime}$ increase during the pedestal recovery, the density dependence of the diamagnetic drift is likely to be weak compared with the temperature dependence. However, improved edge measurements are required to confirm this.

It cannot be excluded that a spin-up of the poloidal plasma rotation $\left(v_{\theta}\right)$ at the edge could also contribute to the observed WB frequency behaviour, but measurement of $v_{\theta}$ is difficult. Neoclassical theory predicts the poloidal rotation of the main ion species to be coupled to the ion temperature gradient $[18,19]$, and therefore $v_{\theta}$ could evolve in time in a manner similar to that of the ion diamagnetic drift. The sign of $v_{\theta}$ depends on the ion collisionality. For the range of collisionalities relevant here $\left(0.05 \lesssim v_{i}^{*} \lesssim 5\right)$, the poloidal rotation is predicted to be in the direction of the electron diamagnetic drift.

The frequency of the WB modes is influenced by the level of gas fuelling. Figure 4 shows a set of discharges with different gas fuelling levels. With increasing gas puff the spectral distribution of WB modes becomes narrower and evolves towards lower frequencies. This behaviour is consistent with the gradually lowered edge temperature. When fuelling levels are low, the individual WB bands are usually well separated in frequency and thus clearly discernable in spectrograms (see also figures 1 and 3). With increasing fuelling the WB band frequencies tend to become closer to each other, and at sufficiently high fuelling rates, they can merge into a broader band of WB activity where the individual WB bands composing it are

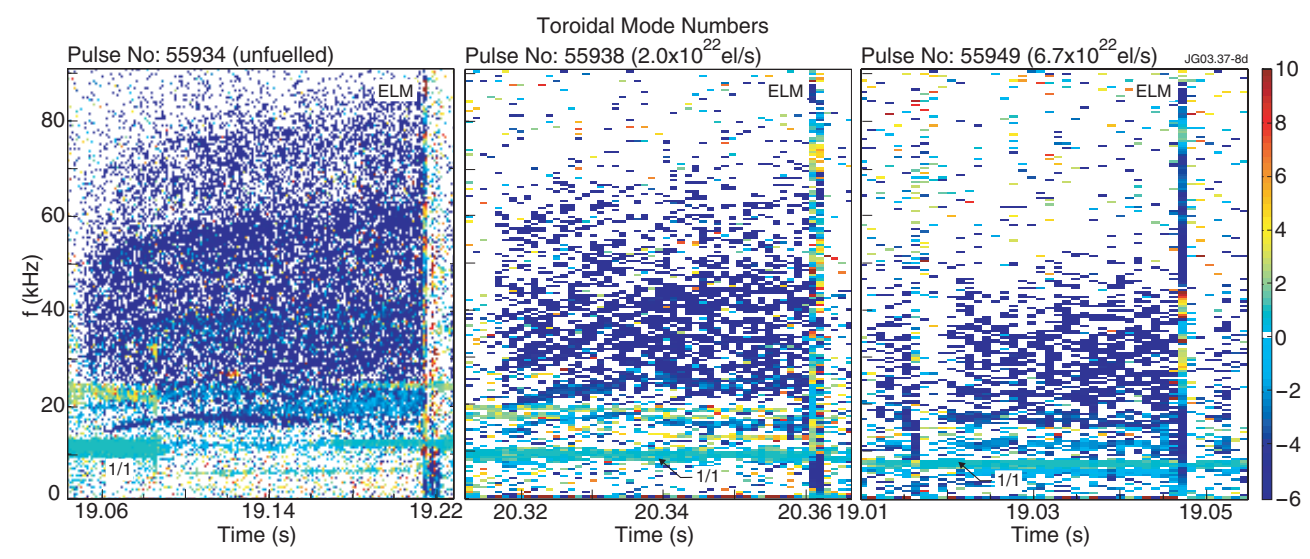

Figure 4. WB modes in a gas puff scan (at low triangularity). Each plot depicts a single ELM period. With increasing gas fuelling (from left to right) the spectral distribution of WB modes becomes narrower and evolves towards lower frequencies. This frequency behaviour is consistent with the gradually lowered edge temperature due to the gas puffing. At high gas rates the WB bands usually tend to occur only around $10-40 \mathrm{kHz}$. The green-coloured modes around $10 \mathrm{kHz}$ are sawtooth precursors. 
difficult to discern any more. At high gas rates the WB activity typically occurs only around $10-40 \mathrm{kHz}$. The time evolution of the edge electron temperature between ELMs does also change with fuelling. The temperature drop per ELM decreases with increasing edge density, and it is sometimes observed that $T_{\mathrm{e}}$ saturates even at an early stage of the ELM period. In that case the WB frequency does not change much, or even remains constant, between ELMs.

WB modes have never been observed in L-mode, but it is difficult to exclude their existence definitely. Due to the absence of a strong edge gradient and its associated diamagnetic drift, very low WB rotation frequencies can be expected, and this would impose inherent difficulties on the detection of the WB modes. So far WB modes have only been observed in situations where a sufficiently developed H-mode pedestal is present. In particular, in an ELM-free or type-I ELMy H-mode, the WB modes are basically always encountered. In situations where the pedestal is present but less pronounced, as in the phase shortly after a type-I ELM, or typically also in a type-III ELM regime, WB modes are often not detected. This suggests that the presence of an H-mode barrier is a necessary (but not a sufficient) condition to destabilize the WB modes.

Figure 5 shows a magnetic spectrogram for a standard type-I ELMy-H discharge, fuelled with $2.3 \times 10^{22} \mathrm{el} \mathrm{s}^{-1}$, showing a time window comprising six type-I ELMs. The colour map of the spectrogram is chosen to change colour only in a narrow range of amplitudes around that of the WB modes. The spectrogram has further been corrected for the frequency dependence of the magnetically measured mode amplitudes. The numbers to the right of the colour scale of the spectrogram denote the $\log _{10}$ of amplitude. Also shown are the traces of the electron temperature near the pedestal top, measured through ECE, and the line averaged edge density, measured by a far infrared (FIR) interferometer [20]. Due to the geometric layout of the interferometer cord used (it views the plasma vertically on the LFS, cutting the midplane $\approx 10 \mathrm{~cm}$ inside the separatrix), the line averaged density measured by this cord is effectively a good surrogate measurement for the density near the pedestal top. The bottom plot in the figure shows the inner and outer divertor $\mathrm{D}_{\alpha}$ signals. The time between two consecutive ELMs, $\Delta t_{\mathrm{ELM}}$, varies greatly from ELM to ELM. The spectrogram also shows that different levels of WB activity are observed in each inter-ELM period. This is further exemplified in figure 6(a), where the time averaged power spectra of the inter-ELM activity prior to the fourth $\left(\Delta t_{\mathrm{ELM}}=62 \mathrm{~ms}\right)$ and the sixth $\left(\Delta t_{\mathrm{ELM}}=24 \mathrm{~ms}\right)$ ELMs are compared. Prior to the fourth ELM, a higher WB activity is observed. For each ELM interval the level of WB activity can be quantified by averaging the individual power spectra over time and integrating the resulting averaged power spectra over the frequency range of the WB modes. In figure $6(b)$ the result of such an analysis is shown, plotted against $\Delta t_{\mathrm{ELM}}$. The analysis has been performed over the whole stationary phase of the discharge, and each circle in the plot corresponds to one interELM period. The WB amplitude and the time, $\Delta t_{\mathrm{ELM}}$, are clearly correlated. The causality of events is however unclear: it is not clear whether the WB modes reach higher amplitudes in the longer inter-ELM intervals simply because the ELM interrupts their growth at a later time or whether strong WB mode activity is responsible for an extension of the ELM period. The diamonds shown in figure $6(b)$ denote the energy loss per ELM $\left(\Delta W_{\mathrm{ELM}}\right)$, recorded during the fast acquisition time window of the diamagnetic loop. The energy loss per ELM is not seen to vary with $\Delta t_{\mathrm{ELM}}$, which implies that the variation in $\Delta t_{\mathrm{ELM}}$ must have a different cause. Also, the input power, $P_{\text {heat }}$, and the radiated power, $P_{\text {rad }}$, keep constant over the time interval analysed (15.5 MW and 7 MW, respectively).

Divertor diagnostics have been examined to gain further insight. In the bottom part of figure 5 the inner and outer divertor $\mathrm{D}_{\alpha}$ signals are shown. A fast spiky activity is observed between the ELMs, which is more pronounced on the inner than on the outer divertor signal. The same activity is observed on the signals of the divertor probes located near the strike 

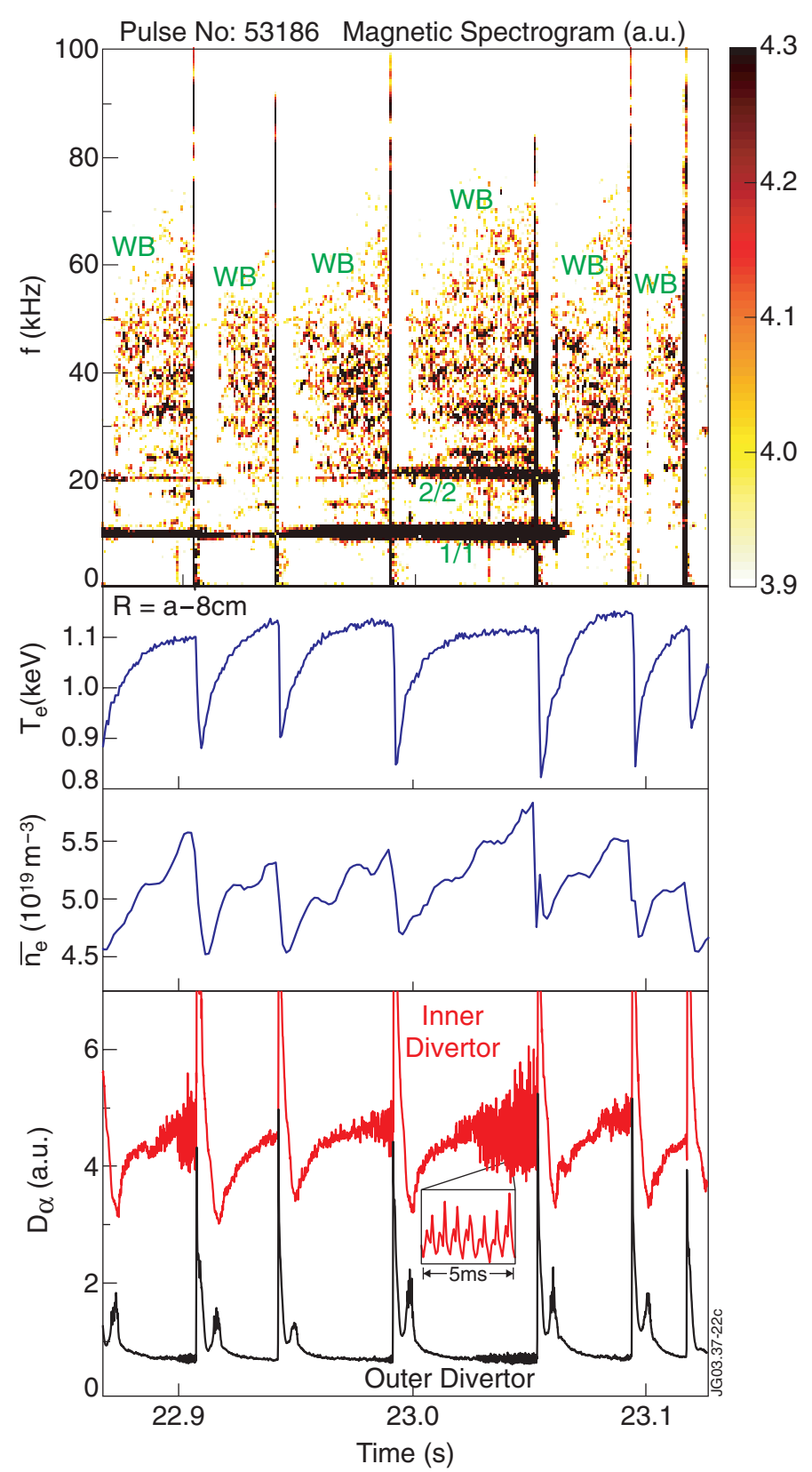

Figure 5. Magnetic spectrogram for a time interval with different levels of WB activity between ELMs, together with the trace of an ECE channel resonant near pedestal top and the line averaged edge density (measured by an FIR interferometer). The bottom plot shows the corresponding divertor $\mathrm{D}_{\alpha}$ signals for the inner and the outer divertors. The numbers given in the colour bar of the spectrogram are in a.u.

points (not shown here). The activity is composed of small bursts with a repetition rate of a few kilohertz. As has been noted above, the WB amplitude has been estimated to fluctuate on a similar timescale. However, it has not been possible to establish from the data whether the observed activity does indeed originate from the WB modes. While there are many discharges 

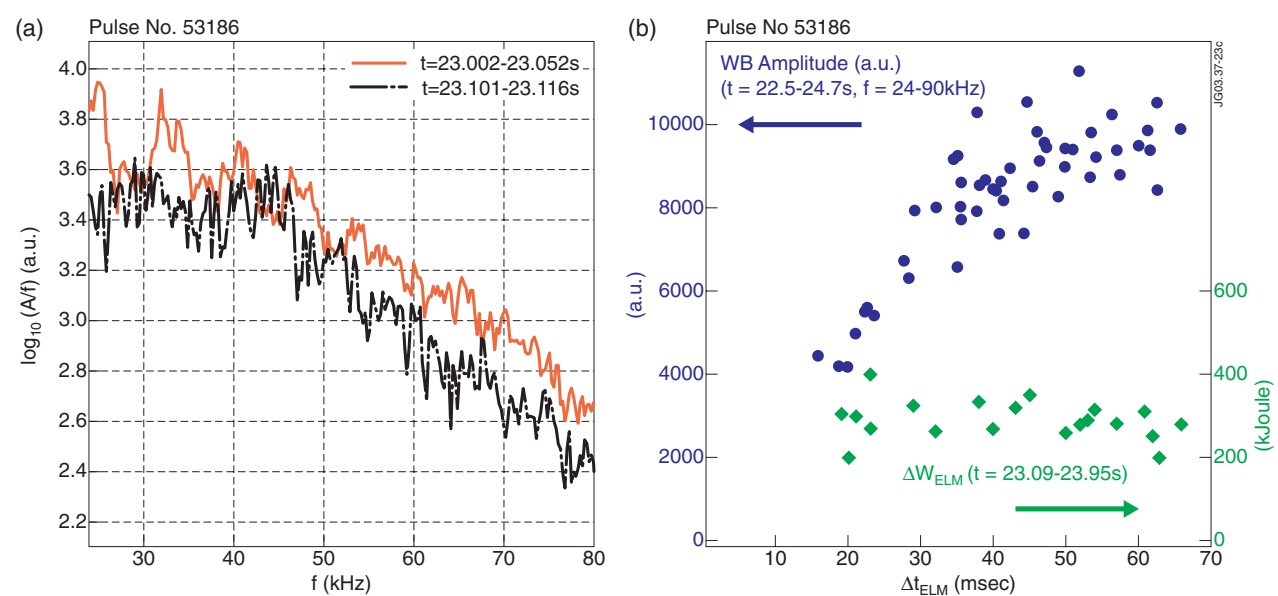

Figure 6. (a) Comparative plot of the time averaged power spectra (at WB frequencies) for the ELM intervals prior to the fourth ELM (-) and the sixth ELM (- - - ) of figure 5, showing a larger overall level of WB mode activity in the former case. (b) Time averaged, frequency integrated WB amplitude ( versus the time $\Delta t_{\mathrm{ELM}}$ between two consecutive ELMs, for the same discharge. The analysis has been performed during the stationary phase of the discharge (from 22.5 to $24.7 \mathrm{~s}$ ). Each circle corresponds to an ELM period. The WB amplitude has been integrated from 24 to $90 \mathrm{kHz}$, thus avoiding the core MHD activity (mainly $1 / 1$ and $2 / 2$ modes), which happens at lower frequencies. Increasing WB amplitude correlates with increasing $\Delta t_{\mathrm{ELM}}$. Also shown is the energy loss per ELM, $\Delta W_{\mathrm{ELM}}(\downarrow)$, determined during the fast acquisition window of the diamagnetic loop (from 23.09 to $23.95 \mathrm{~s}$ ). $\Delta W_{\mathrm{ELM}}$ does not increase with $\Delta t_{\mathrm{ELM}}$.

where the magnitude of the fast inter-ELMD $\mathrm{D}_{\alpha}$ activity is observed to correlate well with the WB mode amplitude, other discharges have been found where this correlation is less convincing. The analysis is further hampered by the strong dependence of the diagnostics' response to the divertor conditions.

Figure 7 shows the time traces for 19 consecutive inter-ELM periods during the stationary phase of discharge 53186, showing the evolution of the time derivative of the edge electron temperature measured near the pedestal top (determined via ECE) relative to the level of WB activity (integrated over frequency from 24 to $90 \mathrm{kHz}$ ). Each curve illustrates the time evolution of the two quantities during one inter-ELM period. The ELM period starts in the lower right part of the plot (low WB amplitude, high $\mathrm{d} T_{\mathrm{e}} / \mathrm{d} t$ ) and evolves towards the upper left corner (high WB amplitude, low $\mathrm{d} T_{\mathrm{e}} / \mathrm{d} t$ ). The curves follow a narrow band in configuration space, independent of the ELM period, indicating the existence of a correlation between WB amplitude and rate of pedestal electron temperature increase. The analogous analysis for the time-derivative of the edge density (relative to the WB mode amplitude) is hampered by an oscillation with $\approx 50 \mathrm{~Hz}$ present on the interferometer signal. The oscillation is regularly encountered on this signal, although with varying degree of accentuation, and is not believed to be real. Its origin is not fully understood yet. The oscillation is clearly recognizable in the density trace shown in figure 5. Without this oscillation, it can be discerned from figure 5 that, unlike for the edge temperature, the rate of increase of edge density does not change greatly throughout an ELM period (this is most clearly seen in the ELM period prior to the fourth ELM). This implies that the edge density cannot (or at least not largely) be affected by the WB mode amplitude. Thus, in contrast to the edge electron temperature, no correlation is found between WB mode amplitude and time-derivative of the edge density.

The strongest evidence obtained so far for the active involvement of WB modes in the plasma edge dynamics comes from discharges in which sharp distinct transitions in WB mode 


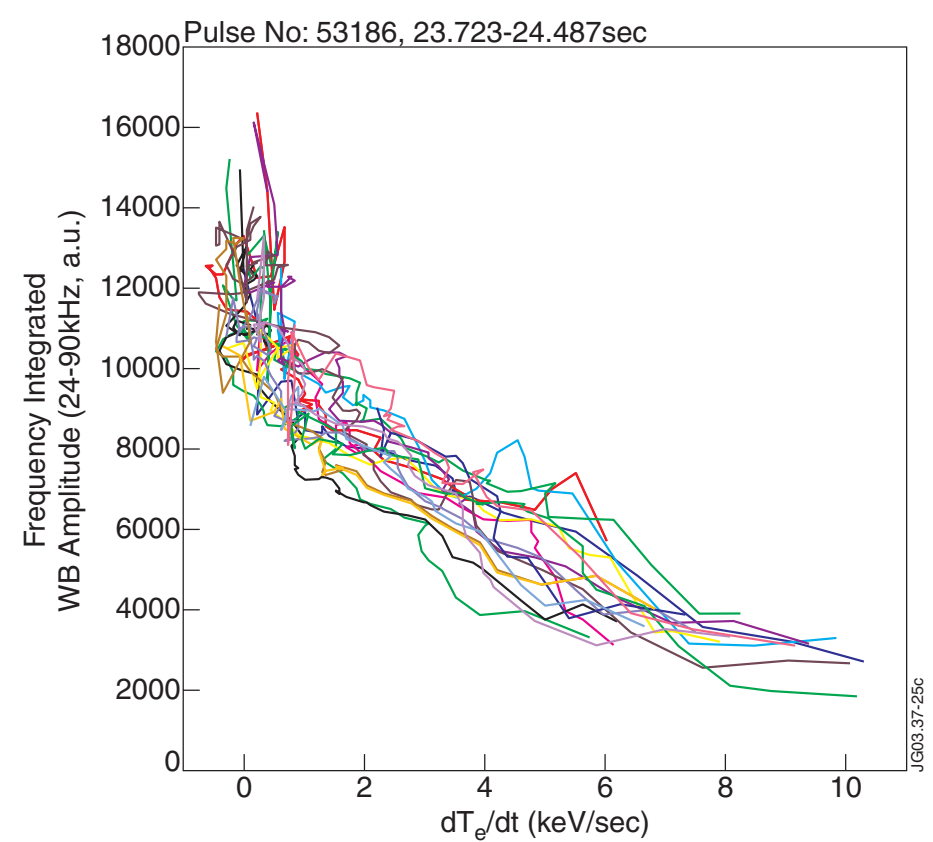

Figure 7. Time traces for 19 consecutive inter-ELM periods during the stationary phase of discharge 53186 (cf figure 5), showing the evolution of the time-derivative of the edge electron temperature measured near the pedestal top (determined via ECE) relative to the level of WB activity (integrated over frequency from 24 to $90 \mathrm{kHz}$ ). Each curve illustrates the time evolution of the two quantities during one inter-ELM period. The ELM period starts in the lower right part of the plot (low WB amplitude, high $\mathrm{d} T_{\mathrm{e}} / \mathrm{d} t$ ), and evolves towards the upper left corner (high WB amplitude, low $\left.\mathrm{d} T_{\mathrm{e}} / \mathrm{d} t\right)$. The curves follow a narrow band in configuration space, independent of the ELM period, indicating a correlation between WB amplitude and rate of pedestal electron temperature increase.

amplitude are encountered. Figure 8 shows the time evolution of the WB mode activity during the ELM-free high performance phase of a hot ion $\mathrm{H}$-mode discharge. A magnetic spectrogram $(a)$ and the corresponding spectrum of $n$-numbers $(b)$ are shown, together with the time traces of the core $(c)$ and edge $(d)$ electron temperature evolution, measured by means of ECE. The bottom plot $(e)$ shows the line averaged edge density measured by the interferometer. In the example shown, three distinct phases can be discerned: during the first phase (up to $t=14 \mathrm{~s}$ ) the WB modes grow slowly, maintaining a modest overall amplitude. The edge temperature grows steadily during this phase. At $t=14 \mathrm{~s}$ a sudden increase in the level of WB activity is observed (second phase), and the growth of the edge electron temperature stops sharply. During this phase, $T_{\mathrm{e}}$ at the pedestal maintains a constant value until $t=14.33 \mathrm{~s}$. At $t=14.33 \mathrm{~s}$, the arrival of an abnormally large sawtooth crash heat pulse at the plasma edge causes a sudden increase in the edge electron temperature, and for reasons that will be further discussed in the conclusions section, the WB mode activity subsequently nearly disappears. From then on the edge electron temperature begins to rise again steadily. Examples like this demonstrate that the WB modes have a regulating effect on the increase of edge temperature. The effect seems not to be restricted to the pedestal top. In discharges with good ECE coverage of the pedestal radii, the effect has also been observed on ECE channels, measuring further outwards in the pedestal region of steep gradients. The only plausible explanation is that WB modes are responsible for a continuous loss of energy into the scrape-off layer (SOL) between ELMs. The existence of a link between the WB mode amplitude and the rate of edge pedestal 
Pulse No. 42840 :

(a) 80 Magnetic Spectrogram

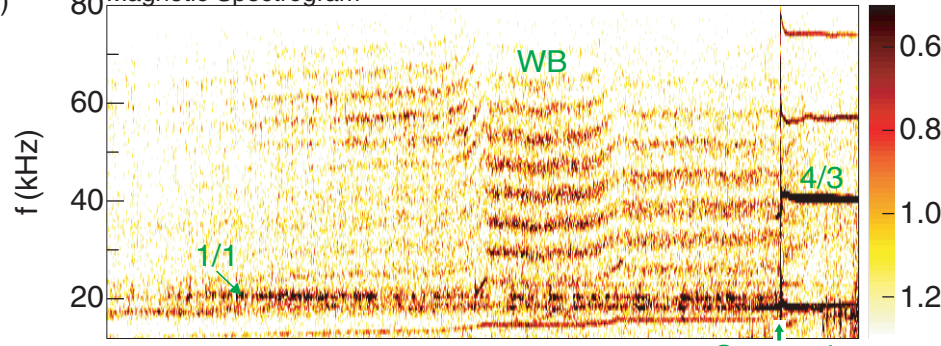

(b)

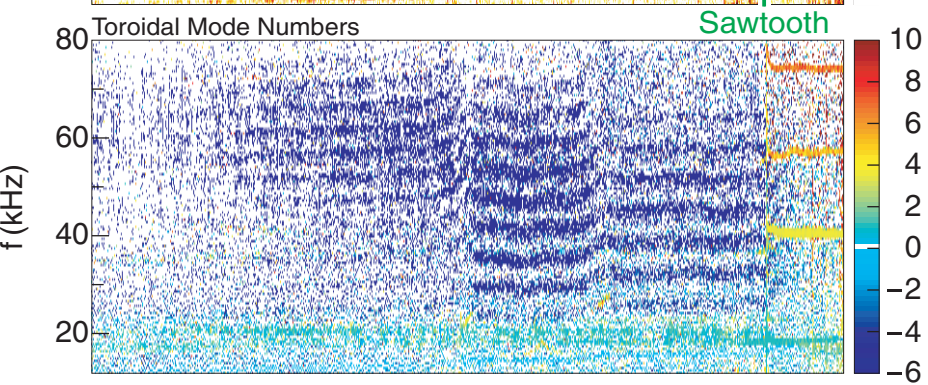

(c)

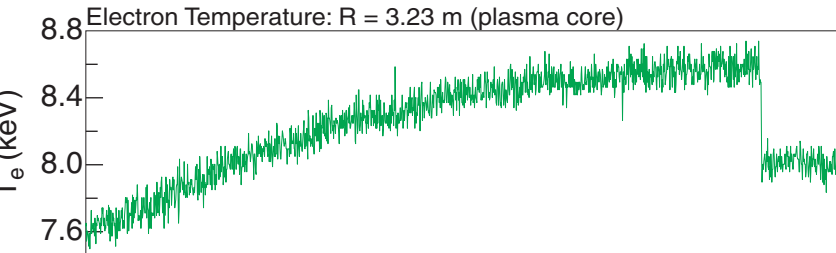

(d) $\quad 4.6$ Electron Temperature: $\mathrm{R}=3.80 \mathrm{~m}$ (pedestal top)

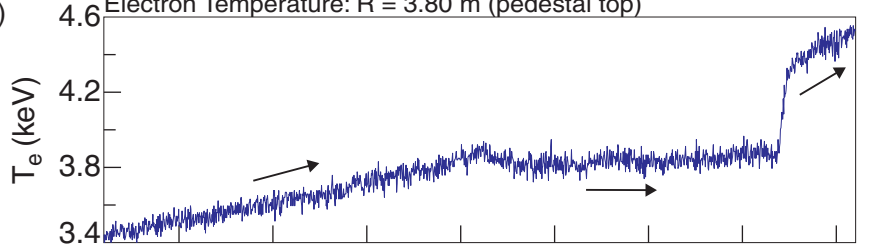

(e)

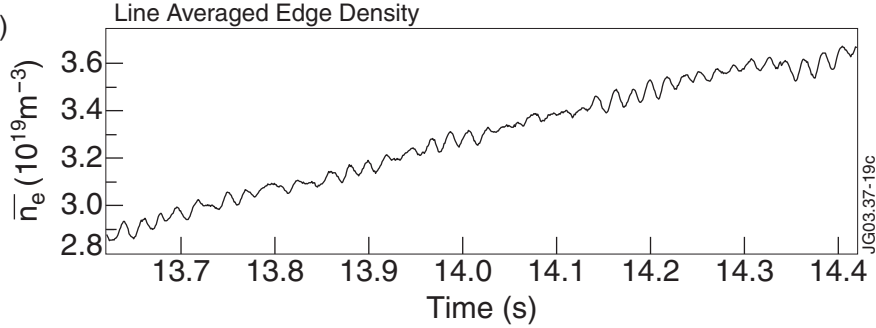

Figure 8. Magnetic spectrogram $(a)$ and spectrum of toroidal mode numbers $(b)$ showing the time evolution of the WB mode activity during the ELM-free high performance phase of a hot ion $\mathrm{H}$-mode discharge. The other figures give the time evolution of the electron temperature in the plasma core $(c)$ near the pedestal shoulder $(d)$ as well as the line averaged edge density $(e)$. Three phases can be discerned: during the first phase (up to $t=14 \mathrm{~s}$ ) the WB modes grow slowly, maintaining modest amplitude. The edge temperature grows steadily during this phase. At $t=14 \mathrm{~s}$ a sudden increase in the level of WB activity is observed (second phase), and the growth of the edge electron temperature stops sharply. During this phase, $T_{\mathrm{e}}$ at the pedestal maintains a constant value until $t=14.33 \mathrm{~s}$. At $t=14.33 \mathrm{~s}$, the arrival of a sawtooth crash heat pulse causes a sudden increase in the edge electron temperature. After the sawtooth crash, the WB mode activity nearly disappears, and the edge electron temperature rises again steadily. The sawtooth crash also triggers core MHD activity (e.g. a 4/3 mode). 
build-up could be established in numerous discharges. Some further examples will be shown in the next section.

\section{WB modes and type-I ELM precursors}

Some of the features of the type-I ELM precursor modes have been outlined in the previous section (the reader is referred to [8] for more details). It has been mentioned that long-lived precursors are often seen to grow and shrink repeatedly before an ELM. Such a case is shown in figure 9, where in addition to the WB modes (again in blue), a yellow-coloured $(n=+4)$ type-I ELM precursor occurs around $15 \mathrm{kHz}$. The precursor starts a long time before the ELM crash, which happens at $21.68 \mathrm{~s}$. A closer examination (zoom view of figure 9) reveals that the precursor is continuously appearing and disappearing again. The remarkable feature is, however, that at times where the precursor occurs the WB activity is seen to vanish. This is illustrated in the bottom plot of figure 9 , where the time-evolution of the frequency integrated amplitude for both the ELM precursor mode and the WB modes are shown. It is evident that the amplitude of the WB modes is in counter-phase to the amplitude of the ELM precursor mode. An exclusive interaction between the two modes must exist. The physical grounds for this interaction have not been yet identified. In general, the order of events observed is that after an ELM crash first the WB modes reappear, and later on usually one or more type-I ELM precursors set in. Coinciding with the onset of the ELM precursor modes, some of the WB bands (or, as in figure 9, all of them) are seen to become weaker. The possibility that a weakening of the WB modes allows the ELM precursors to grow can be excluded by discharges in which the WB modes do weaken but the ELM precursors do not immediately start to grow (see, e.g. figure 8). Thus, the causality appears to be that the growth of ELM precursor modes weakens the WB modes, and not the reverse.

Sometimes not all the WB bands become inhibited. The frequency of the remaining WB activity is then often observed to increase with precursor onset. This is best discerned if the WB frequency has reached a steady value prior to the precursor appearance, and an example is shown in figure 10. On the spectrum of toroidal mode numbers two diffuse WB bands can be recognized around $\sim 36$ and $45-50 \mathrm{kHz}$ (labelled WB1 and WB2, respectively). Both WB bands maintain a roughly constant frequency until the onset of ELM precursors at $16.88 \mathrm{~s}$ (the dark red-coloured modes at $5-10 \mathrm{kHz}, n=9-10)$. WB2 is inhibited by their occurrence, as shown in the bottom plot of the figure. The amplitude shown in that plot has been obtained by integrating, at each time step of the Fourier analysis, over an $8 \mathrm{kHz}$ wide frequency band whose frequency tracks the frequency of WB2. If, in the bottom plot, one regards the level right after the ELM crash as the WB-free background, then the precursor has led to a drop in amplitude of about $70 \%$. A similar analysis has been carried out for the amplitude of WB1 (not shown here). It is obscured by the $3 / 3$ component of the internal kink, which oscillates in amplitude and coincides with the frequency of WB1, but it is believed that the amplitude of WB1 has not been greatly affected by the ELM precursors. One can clearly see that the ELM precursors trigger an increase in the frequency of WB1 until interrupted by the ELM at $16.93 \mathrm{~s}$. It is worth recalling from section 2 that a relationship exists between the WB frequency and the electron temperature evolution measured near the pedestal top. Together with the mode number spectrum the corresponding traces of the edge electron temperature and the line averaged edge density are shown, obtained through ECE and interferometry, respectively. The electron temperature evolution resembles the WB frequency, and in particular, it begins to rise (this is highlighted by the arrow) with the ELM precursor appearance. The temperature increase does not originate from a simultaneous decrease in pedestal density. Instead, the density continues to increase and seems not to be greatly affected. Hence, the onset of type-I 


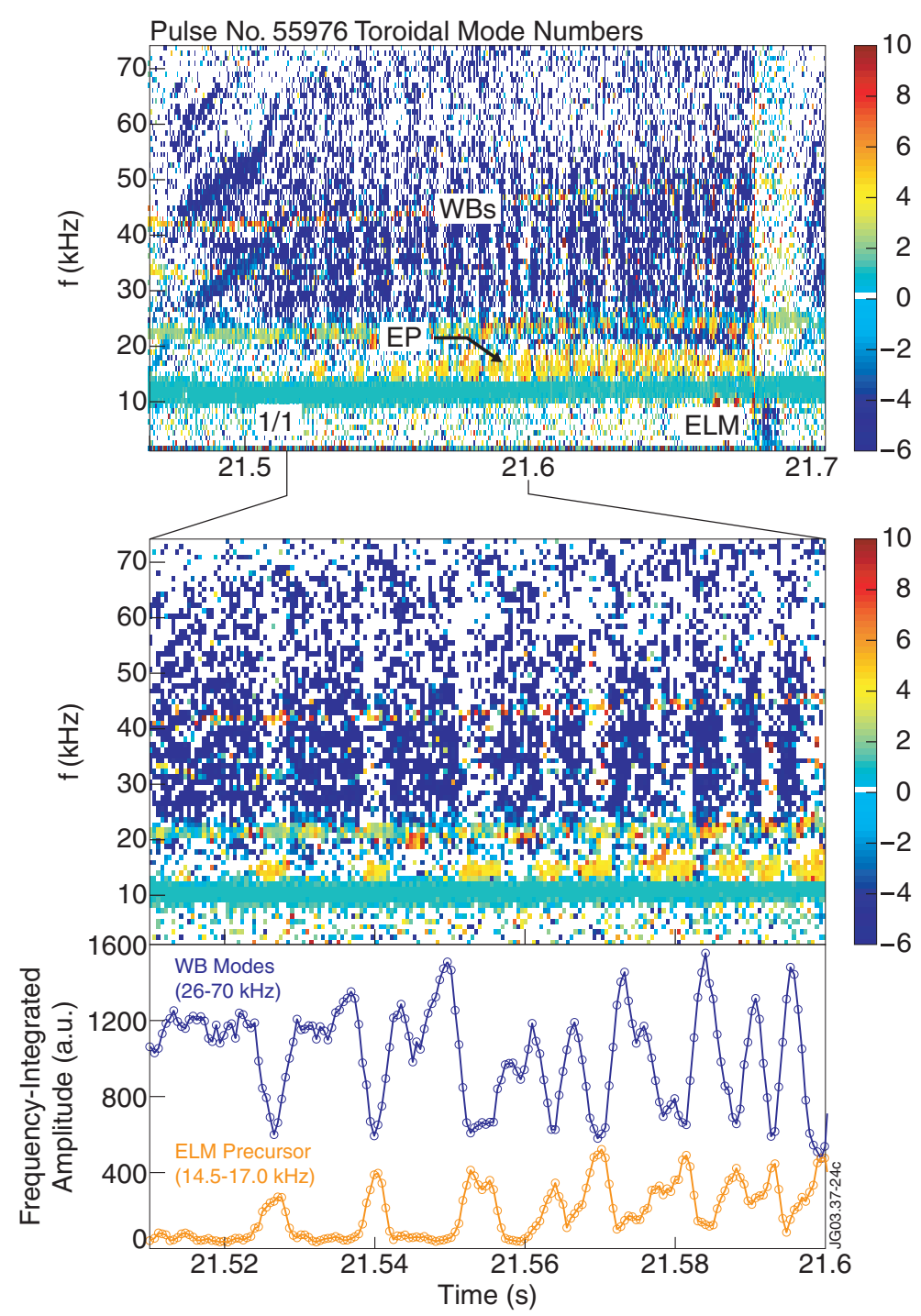

Figure 9. Spectrum of toroidal mode numbers for an inter-ELM period, and a zoom view, showing the WB modes (in blue) and a yellow-coloured $(n=+4)$ type-I ELM precursor mode around $15 \mathrm{kHz}$. The precursor mode starts a long time before the type-I ELM (which happens at $21.68 \mathrm{~s}$ ). The continuously present green-coloured mode at $10 \mathrm{kHz}$ is a sawtooth precursor. In the bottom plot the corresponding time-evolution of the power spectra (frequency integrated over the range of frequencies relevant for each mode) for both the ELM precursor mode and the WB modes are shown. The circles on the curves correspond to one time-slice of the Fourier analysis. To smoothen the curves, the values obtained for each time-slice have been averaged with the values for the previous and the next time-slices. The amplitude of the WB modes is in counter-phase to the amplitude of the ELM precursor mode.

ELM precursors has indirectly led, through a WB mode weakening, to an acceleration of the build-up of the electron pedestal pressure, though the mechanism for this remains unclear. Due to diagnostic limitations no comparable data are available to study the temperature evolution of the ion species. It is however expected that collisional energy exchange with the electrons will result in a similar behaviour for the ions. 


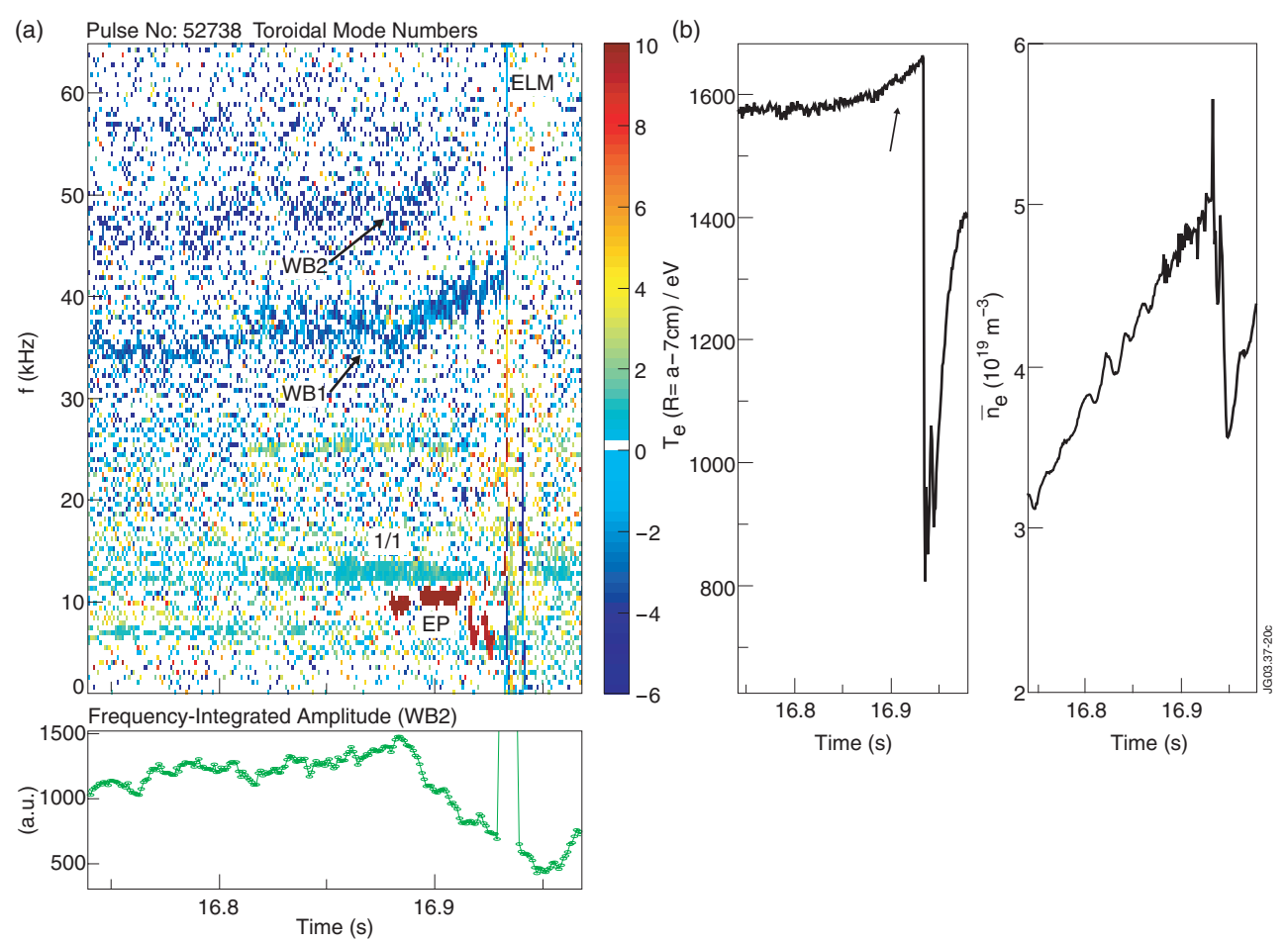

Figure 10. (a) Spectrum of toroidal mode numbers highlighting two diffuse WB bands: one around $36 \mathrm{kHz}$ (labelled with WB1), in light blue, and a further even more diffuse WB band at $45-50 \mathrm{kHz}$, labelled with WB2. Both WB bands maintain a roughly constant frequency until the onset of ELM precursors at $16.88 \mathrm{~s}$ (the dark red-coloured modes $(n=9-10)$ at $5-10 \mathrm{kHz})$. WB2 is inhibited by their occurrence, as shown in the bottom plot. The ELM precursors further trigger an increase in the frequency of the remaining WB band WB1 until interrupted by the ELM at $16.93 \mathrm{~s}$. (b) Corresponding traces of electron temperature measured near the pedestal top, and of the line averaged edge density. The temperature evolution resembles the WB frequency and begins to increase at precursor onset (this is highlighted by the arrow). In contrast, the density build-up remains seemingly unaffected.

From figure 10 one can discern that the edge electron pressure has been constantly increasing prior to the onset of the ELM precursor, through the increasing edge density. Clearly, the pressure observed prior to the ELM onset would have also been reached in the absence of ELM precursors, though at a slightly later time. Therefore, it is expected that the ELM would have been triggered in any case, also in the absence of ELM precursors. The real value of this kind of example lies in the valuable information that it provides about the operational point of the pedestal in terms of edge current density and edge pressure gradient, and its actual position relative to the kink- and ballooning-stability boundaries. This will be further addressed in section 5 .

It has to be underlined that this pattern of events (ELM precursor occurrence and a reduction in the WB mode amplitude, and a further increase in the edge electron temperature) is not restricted to the above example but is a robust phenomenon that has been observed repeatedly in JET discharges. Figure 11 shows the magnetic spectrogram and ECE signals corresponding to the spectrum of $n$-numbers used for the introductory example of figure 1 . As seen on the spectrogram, the long-lived ( $\sim 25 \mathrm{~ms})$ precursor around $18 \mathrm{kHz}$ prior to the second ELM inhibits a WB band at $25 \mathrm{kHz}$ (the same happens prior to the third ELM, though the $2 / 2$ mode 

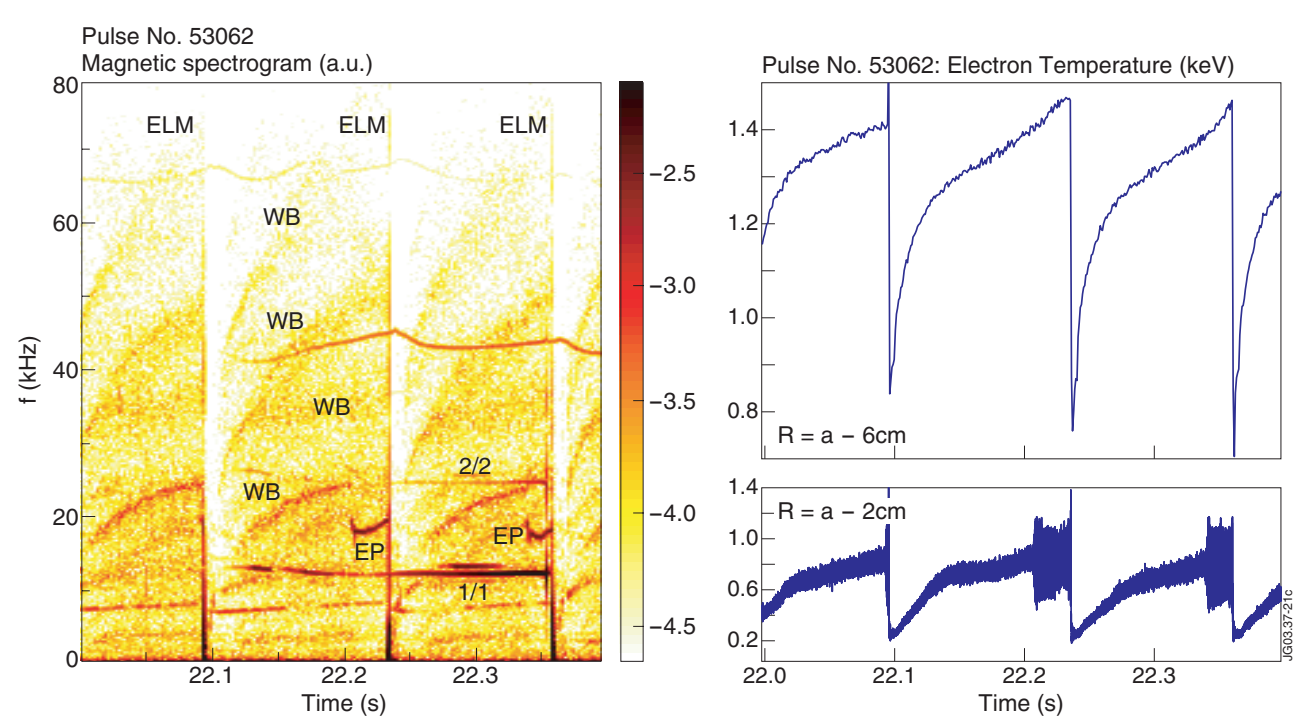

Figure 11. Magnetic spectrogram from a coil located on the LFS, corresponding to the introductory example shown in figure 1. The long-lived ELM precursor (EP) around $18 \mathrm{kHz}$ prior to the second type-I ELM inhibits the WB band at $\sim 25 \mathrm{kHz}$. (The same happens prior to the third type-I ELM, though the $2 / 2$ mode makes it more difficult to see.) Shown on the right are the signals from two ECE channels: one resonant $6 \mathrm{~cm}$ inside the separatrix (near the pedestal shoulder, top plot) and one resonant $2 \mathrm{~cm}$ inside the separatrix (in the pedestal region of steep gradients, bottom plot). The latter signal is sampled at $250 \mathrm{kHz}$. The precursor oscillations are detected on the channel resonant $2 \mathrm{~cm}$ inside the separatrix. On the channel measuring $6 \mathrm{~cm}$ inside the separatrix a slight but still discernable increase in the build-up of the edge electron temperature is observed in the presence of the ELM precursors. Prior to the first ELM a much shorter-lived ( $\sim 1.5 \mathrm{~ms})$ precursor existed. Due to its shortness that precursor could not affect the evolution of the pedestal build-up significantly.

makes it more difficult to see). Again, the amplitude of the other WB bands seems not to be greatly affected by the ELM precursor. This could be an indication that a WB band has to meet some kind of 'resonance condition' for the inhibition by the ELM precursor to take effect, but the data analysed so far are not yet conclusive in this respect. The precursor oscillations are detected on the fast ECE channel measuring $2 \mathrm{~cm}$ inside the separatrix (in the pedestal region of strong gradients), while on the channel measuring $6 \mathrm{~cm}$ inside the separatrix (near the pedestal top) one can observe a slight but still discernable increase in the slope of the edge electron temperature build-up when the precursors are active. Prior to the first ELM a much shorter-lived $(\sim 1.5 \mathrm{~ms})$ precursor existed. Due to its shortness that precursor could not affect the evolution of the pedestal build-up significantly. The case of short-lived precursors, which is quite often observed, will be further addressed in section 5 .

A general consequence of the above observations is that due to their complex interplay with the WB modes, the onset of ELM precursor modes does not commonly lead to an overall pedestal (and thus confinement) degradation, but rather to an improvement of the pedestal. This finding is certainly counter-intuitive and unexpected. After the onset of ELM precursor modes, the resulting evolution of the pedestal will be a trade-off between the decrease in transport associated with the observed suppression of WB modes and any increase in transport associated with the ELM precursor modes themselves. The commonly observed accelerated build-up of the pedestal pressure implies a greater particle and energy transport associated with the WB modes. In situations in which the WB modes were absent, the onset of ELM precursor modes has not resulted in an accelerated pedestal build-up and could even lead to a pedestal degradation. 
ELM precursors are not the only possible source of WB mode suppression. WB weakening due to an abnormally large sawtooth heat pulse has been already shown in figure 8 . Some further cases have been found in which the WB modes become stabilized 'spontaneously' in the middle of an inter-ELM period with no identified cause and, in particular, with no precursors having been detected.

\section{WB modes and type-II ELMs}

The 'normal' ELM frequency behaviour observed in low triangularity discharges is that the type-I ELM frequency increases with increasing level of gas puffing. Three effects contribute to this behaviour: first, the pressure at the top of the pedestal, $p_{\text {ped }}$, and therefore the energy content of the pedestal, which is given by $3 / 2 \times p_{\text {ped }} \times V_{\mathrm{p}}$ (where $V_{\mathrm{p}}$ is the total plasma volume) [21], is observed to decrease with increasing density. Therefore, at fixed input power, less time is needed for the pedestal to reach the observed critical pressure. Second, the energy loss per ELM relative to the pedestal energy content is observed to decrease with increasing density. Third, the recovery of pedestal density between ELMs is observed to become faster with fuelling (the simplest explanation is the increased gas fuelling itself, but changes in inter-ELM transport might play a role as well).

Discharges at high triangularity $(\delta \sim 0.5)$ show a clear deviation from the standard ELM behaviour, with the ELM frequency decreasing for the higher fuelling rates [22-24], as shown in figure 12. In spite of the lowered ELM frequency, the energy loss per ELM does not increase

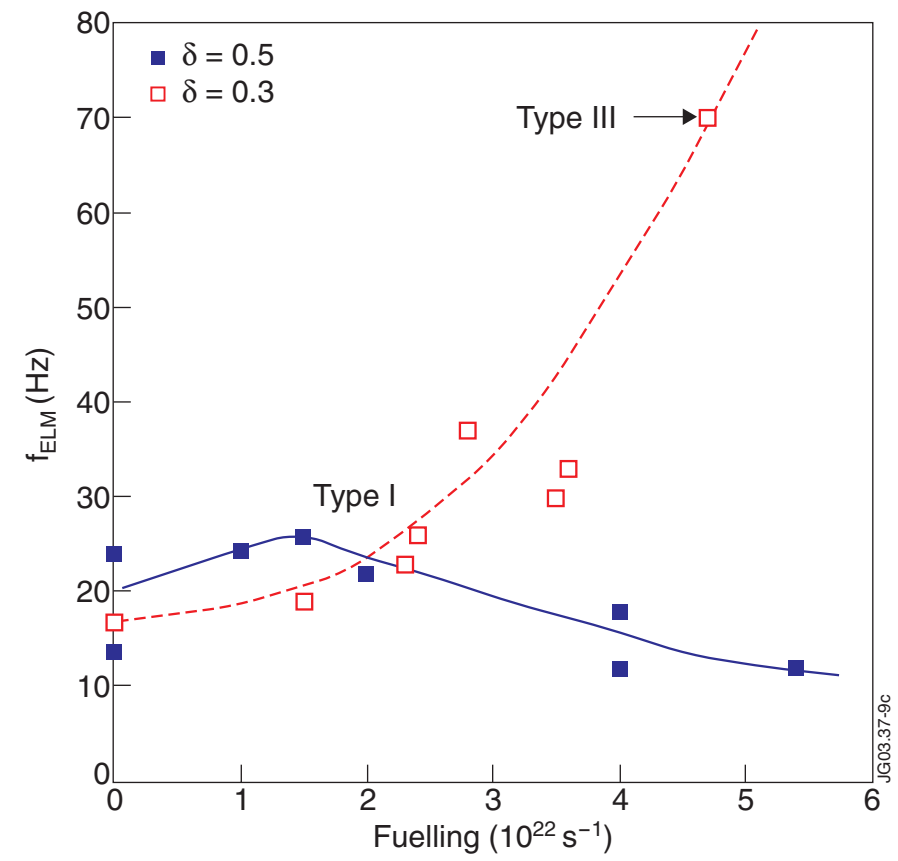

Figure 12. Behaviour of ELM frequency with increasing levels of gas fuelling for two scans performed at low $(\delta=0.3)$ and high $(\delta=0.5)$ plasma triangularity (from [24]). For discharges at high triangularity the ELM frequency decreases for sufficiently high fuelling rates, showing a clear deviation from the standard ELM frequency behaviour. This observation led to the identification of a new regime on JET that has been called the mixed type-I/type-II ELM regime. 
in these cases [22]. Instead, simple power balance considerations demonstrate that the lowered ELM frequency originates from enhanced inter-ELM losses [25]. The anomalous ELM frequency behaviour is accompanied by broadband MHD activity around $30 \mathrm{kHz}$ propagating in the direction of the electron diamagnetic drift, and a grassy signature on the inter-ELM divertor $\mathrm{D}_{\alpha}$ [26]. The similarity of the observations with the type-II ELM regime observed in ASDEX-U [27] suggested the identification of the anomalous ELM regime observed at JET as a mixed type-I/type-II ELM regime [25].

Figure 13 shows the parameter evolution of a typical JET discharge with mixed type-I/type-II ELM regime. The presence of a mixed ELM regime during the gas puff phase is recognizable through the lowered type-I ELM frequency compared with after fuelling switchoff. Figure 14 gives an overview of the MHD activity, and in particular of the WB activity, observed at times with and without the gas fuelling. (The gas puff has been switched off at $\sim 22.5 \mathrm{~s}$.) The WB mode frequencies behave in the expected way, as described in section 2 : during the gas puff the WB modes tend to maintain a constant frequency between ELMs and occur only around $10-40 \mathrm{kHz}$. After switching off the fuelling the WB bands evolve towards higher frequencies, covering a wider spectral range. The enhanced activity seen at $10-40 \mathrm{kHz}$ during the gas puff phase has been associated with the type-I/type-II ELM regime occurrence [26]. It is evident that these are WB modes.
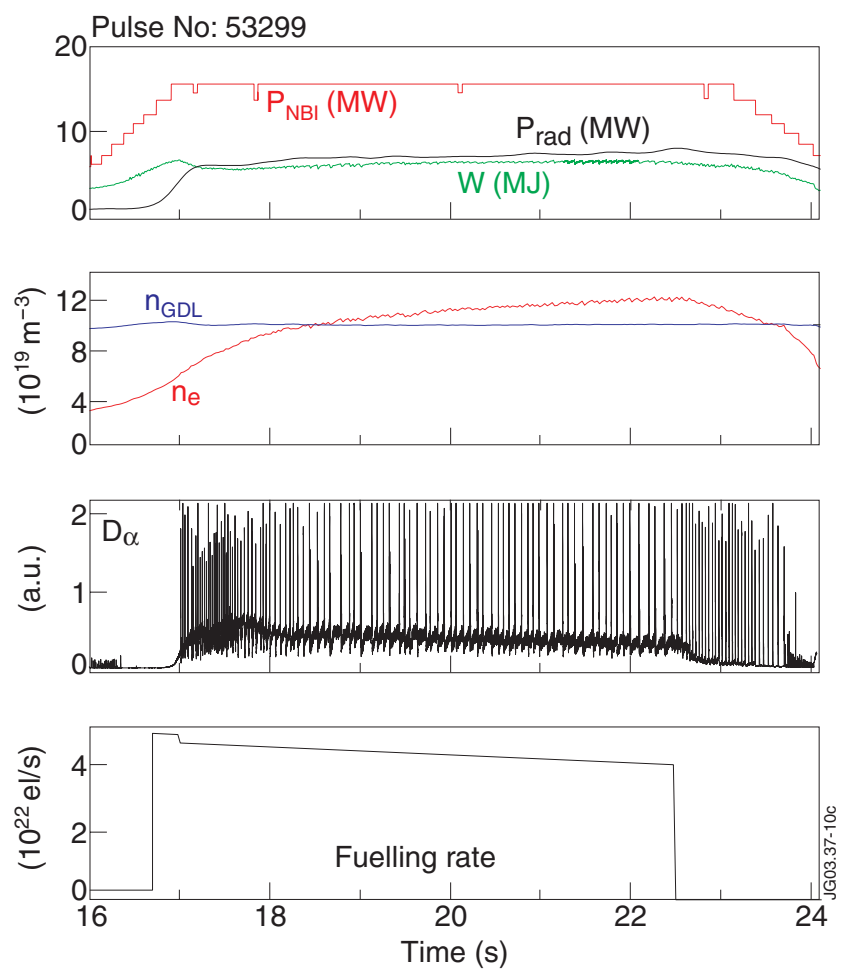

Figure 13. Parameter evolution of a typical JET discharge (2.7 T, $2.5 \mathrm{MA}, \delta \sim 0.5)$ with the mixed type-I/type-II ELM regime. Shown are $(a)$ the NBI power, the plasma energy content, $W$ and the radiated power, $P_{\text {rad }},(b)$ the line averaged density together with the Greenwald density limit, (c) the divertor $D_{\alpha}$ signal, where the large spikes are due to type-I ELMs, and $(d)$ the gas fuelling rate. The presence of a mixed ELM regime during the gas puff phase is recognizable through the lowered type-I ELM frequency while gas fuelling is on. 


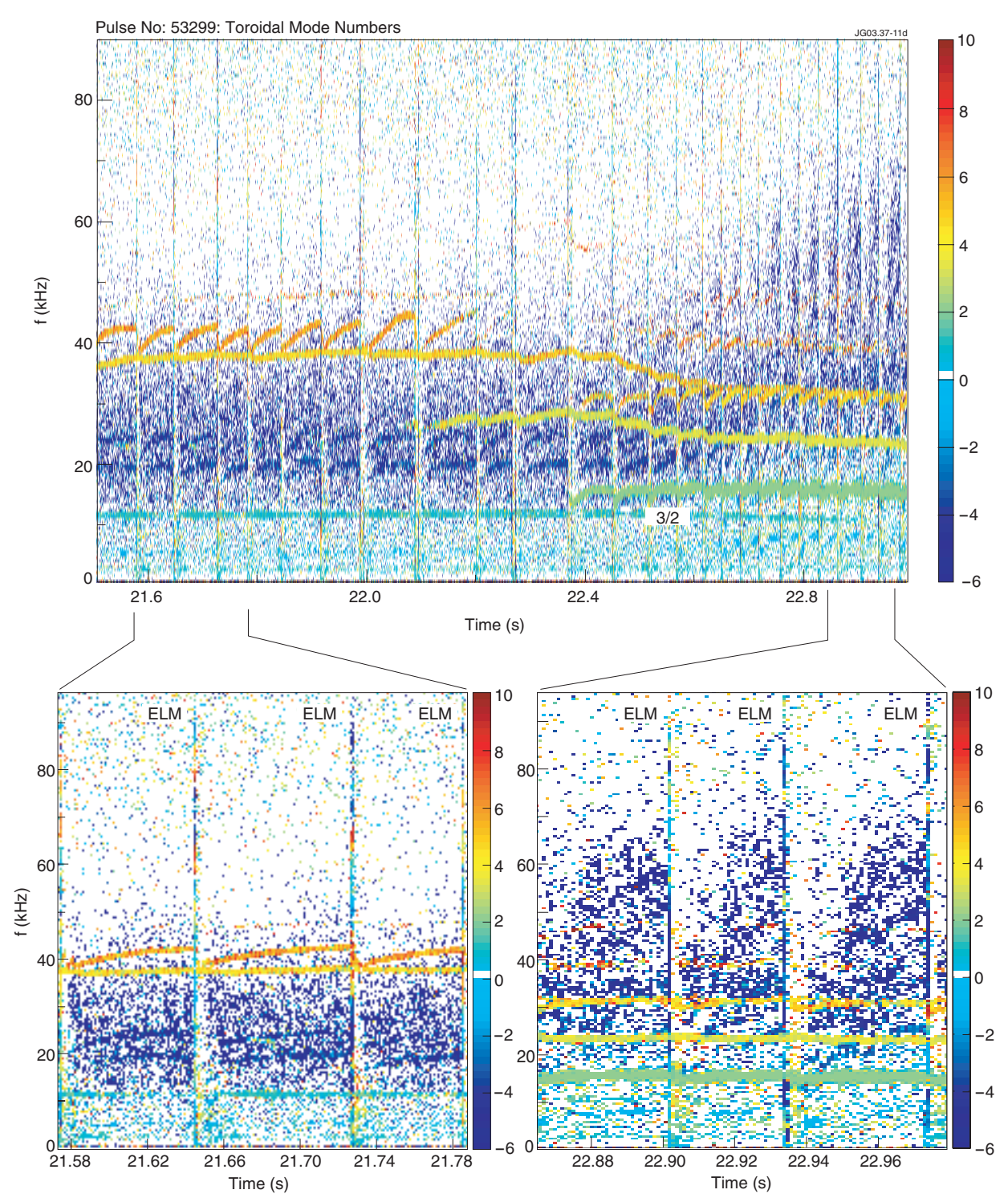

Figure 14. Spectrum of toroidal mode numbers for the same discharge as figure 13, taking closer looks into the WB activity (in blue) in the mixed type-I/type-II ELM regime (during gas puffing) and in the type-I ELM regime (gradually recovered after switching off the gas fuelling at $\sim 22.5 \mathrm{~s}$ ).

Figure 15 shows magnetic spectrograms for two pairs of discharges with different fuelling rates at low and high triangularity. In order to allow comparison, the mapping of amplitudes to colours is the same for each pair of discharges. In the case of low triangularity (figures 15(a) and (b); 2.4 T, 2.4 MA, 15 MW NBI), gas puffing weakens the WB activity, while in high triangularity discharges (figures $15(c)$ and $(d) ; 2.7 \mathrm{~T}, 2.5 \mathrm{MA}, 15 \mathrm{MW} \mathrm{NBI}$ ) the WB activity is clearly enhanced with gas fuelling. The enhancement of WB modes through gas fuelling at high triangularity, and the increase of inter-ELM losses that this is expected to cause, can therefore provide an explanation for the ELM frequency anomaly and the occurrence of the so-called mixed type-I/type-II ELM regime at JET. 

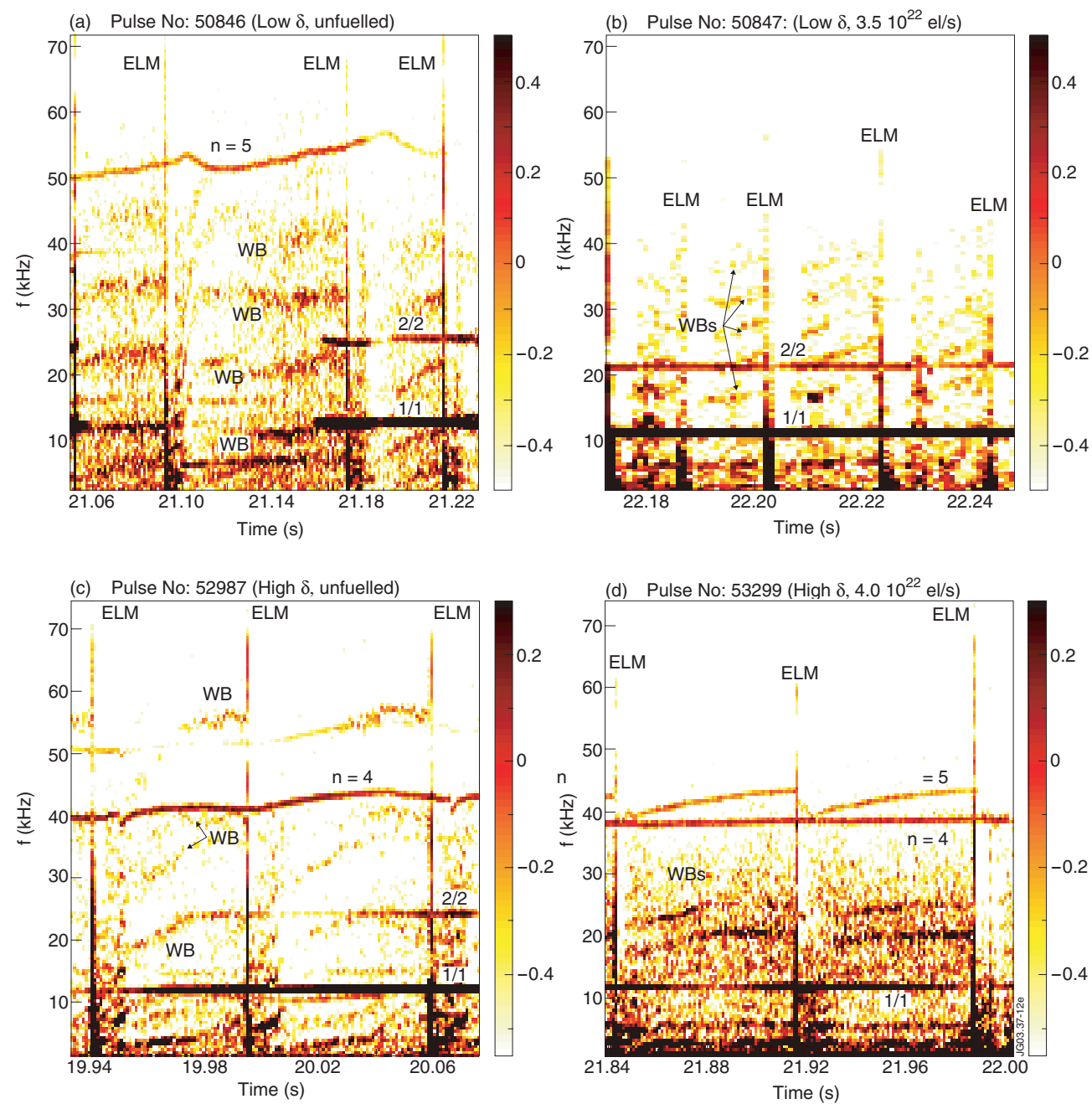

Figure 15. Magnetic spectrograms for two pairs of low $(\delta \sim 0.3)$ and high triangularity $(\delta \sim 0.5)$ discharges with and without strong gas fuelling. At low triangularity, WB modes become weaker with fuelling, whereas at high triangularity it is the opposite case. The colour map for the amplitudes has been kept fixed for each pair to allow comparison. The numbers to the right of the colour scales denote the logarithm $\left(\log _{10}\right)$ of amplitude, and are given in a.u.

WB modes provide the enhanced turbulence identified in $[25,26]$ as a characteristic signature of the mixed type-I/type-II ELM regimes at JET, but it has to be emphasized again that WB modes are also present in regimes regarded as pure type-I ELM regimes. It has been shown in the previous sections that WB modes influence the pedestal dynamics also in the latter regime.

\section{WB modes and the peeling-ballooning cycle}

The peeling-ballooning cycle [6] represents a model for type-I ELMs that has gained wide acceptance and constitutes a promising candidate for understanding ELMs. It is worth briefly 


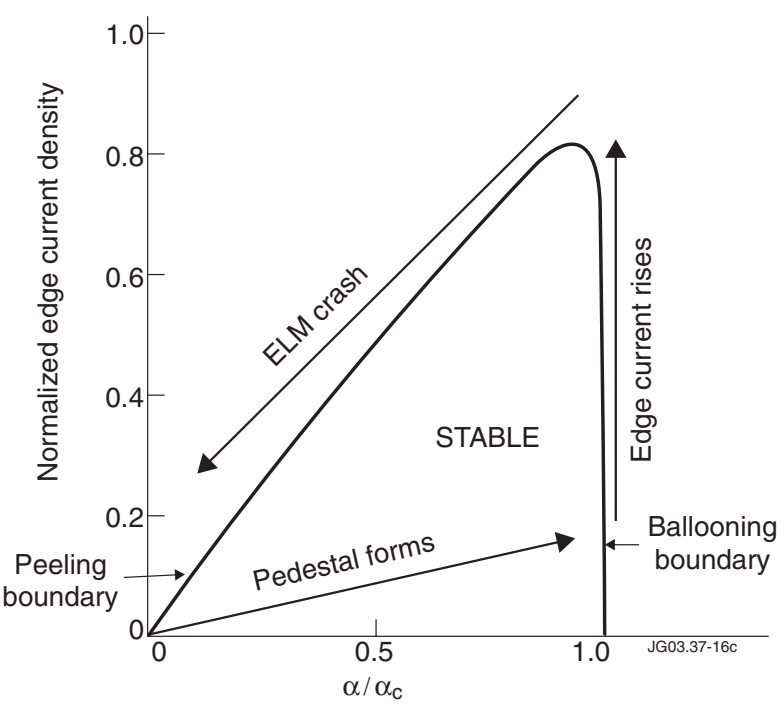

Figure 16. Stability diagram for coupled peeling-ballooning modes in the space of normalized edge current and ballooning stability parameter showing the order of events predicted by the conventional peeling-ballooning cycle (from [3]). Just after the previous ELM crash the plasma edge resides in a state of low pressure and low edge current (lower left corner in diagram). Heating builds up the pedestal pressure gradient on a relatively fast timescale until the ideal ballooning limit is reached, where it is limited. With the edge current gradually building up on a slower (resistive) timescale, the pedestal state moves towards the upper right corner of the stability diagram. The ELM is finally triggered when the peeling stability boundary is crossed, where the onset of peeling (or coupled peeling-ballooning) modes causes a further degradation in confinement and therefore a further destabilization of the modes.

reviewing the order of events predicted by this cycle, which is conceptually depicted in figure 16: just after the previous ELM crash the plasma edge resides in a state of low pressure and low edge current (lower left corner in diagram). Heating builds up the pedestal pressure gradient on a relatively fast timescale until the ideal ballooning limit is reached, where, according to the model, it is held. The peeling-ballooning model assumes that the ideal ballooning instability is a benign instability that does not induce a violent event but rather limits the operational space in a smooth way. In a further step, with the edge current gradually building up on a slower (resistive) timescale, the pedestal state moves towards the upper right corner of the stability diagram. The ELM is finally triggered when the peeling stability boundary is crossed, where the onset of peeling (or coupled peeling-ballooning) modes causes a further degradation in confinement and therefore a further destabilization of the modes.

It is worth mentioning that the model of the peeling-ballooning cycle has been recently expanded by Snyder et al [28] to include further ELM types. Within that model, the new ELM types (type-II ELMs, low density type-III ELMs) are described by cycles with alternative trajectories in the edge current/edge pressure stability diagram.

Comparison with the experimental observations for type-I ELMs made on JET shows some disagreement with the predictions of the peeling-ballooning cycle.

At sufficiently low edge collisionality $\left(v_{\mathrm{e}}^{*} \lesssim 0.3\right.$ ), type-I ELMs are regularly preceded by external kinks (low- $n$ ELM precursors). The kink modes start typically $10-100 \mathrm{~ms}$ before the ELM [8]. Hence, in that case the kink stability limit is reached well in advance of the ELM event. In addition, the precursor growth rate is generally not seen to increase significantly prior to the ELM. This suggests that the external kinks do not finally trigger the ELM. 
Similarly, the kink limit is probably reached well before the type-I ELM, also when higher- $n$ precursors are observed. They commonly precede ELMs in discharges with low to moderate edge electron collisionality (roughly $0.3 \lesssim v_{\mathrm{e}}^{*} \lesssim 2$ ) but are no longer observed in discharges with strong gas puffing, where according to modelling results the high edge collisionality leads to a significant reduction of the edge current and keeps the pedestal away from the kink limit [29]. Hence, higher- $n$ precursors do probably become destabilized at the kink limit or near the top right corner in the stability triangle (which is consistent with peeling-ballooning modes, as proposed in [8]). Higher- $n$ precursors are again observed to start well before the ELM $(\sim 1-50 \mathrm{~ms})$. No rapid increase in their growth rate prior to the ELM is observed, which suggests that they do not trigger it.

The peeling-ballooning model does not take into account the physics of WB modes, which has been shown to play an important role in the pedestal dynamics. Therefore, a modified version of the peeling-ballooning cycle that takes them into account and is consistent with the experimental observations presented here is proposed. The individual steps are conceptually depicted in figure 17, where the WB unstable region has been shaded. Its precise shape is
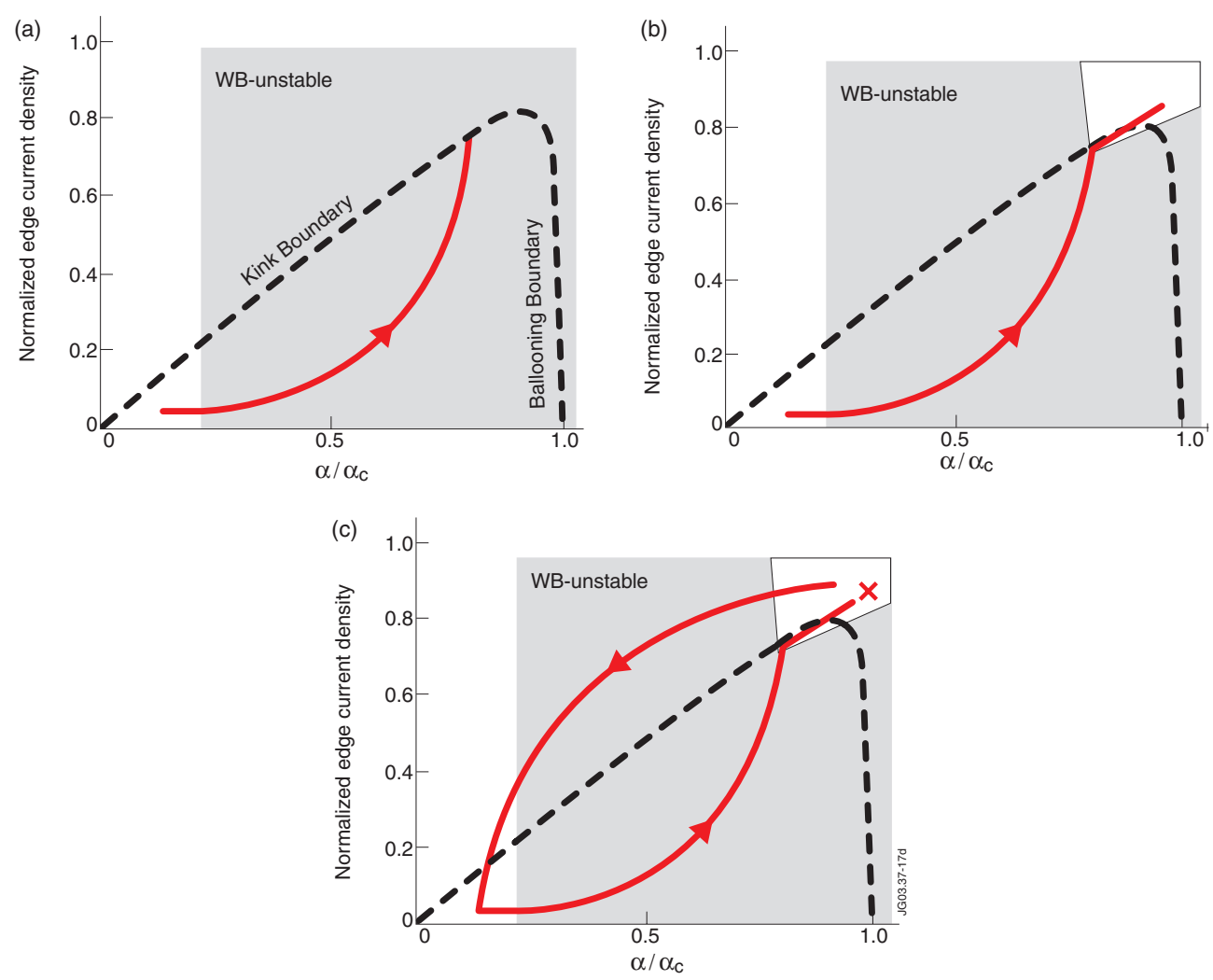

Figure 17. Individual steps of a modified peeling-ballooning cycle that takes into account the WB mode observations: (a) after the previous ELM crash the pedestal pressure initially builds up unperturbed, but with the onset of WB modes and the associated increase in inter-ELM losses, the pressure build-up is slowed down, allowing the edge current to build up on a comparative timescale until the kink limit is reached. (b) ELM precursors become destabilized at the kink limit, and their interaction with WB modes weakens or stabilizes the WB modes, allowing a further build-up of the pedestal pressure along or near the kink limit. (c) The ELM is finally triggered at the ideal ballooning limit, which is now assumed to impose a hard limit. 
not known, but from the observation that WB modes reappear soon after the previous ELM crash, it must be concluded that they can become unstable at a relatively low pedestal pressure (far below the ideal ballooning limit) and low edge current density. Starting at step 1 from a state of low pressure gradient and low edge current after the previous ELM crash, the pedestal pressure initially builds up unperturbed. Then, with the onset of WB modes and the associated increase in inter-ELM losses, the pressure build-up is slowed down, allowing the edge current to build up on a comparative timescale until the kink stability limit is reached. In step 2, ELM precursors become destabilized at the kink limit (the ELM itself is not triggered at this stage because experimentally the ELM precursors are not seen to trigger the ELM). The weakening of WB modes associated with the occurrence of the ELM precursors allows for a further build-up of the pedestal pressure along or near the kink limit. The pedestal may shortly move again into the kink-stable region, where the precursors become stabilized, but with the reappearance of WB modes the kink limit will be reached soon again, leading to the observation of intermittently existing precursors (as in figure 9). If the pressure build-up occurs entirely within the kink-unstable region, the precursors will be continuously present. At this stage the mode numbers of the most unstable precursor modes will eventually change during the pedestal evolution, leading to the occurrence of a sequence of precursors with different mode numbers or the existence of more than one precursor mode at a time (this has been reported in [8]). The ELM is finally triggered when the pedestal reaches the ideal ballooning limit (step 3), which in contrast to the conventional model for the peeling-ballooning cycle, is now assumed to impose a hard limit. In this respect, certain theoretical works [30,31] predict a general explosive feature of the ideal ballooning instability ('detonation') emerging from non-linear effects through the development of fine-scale substructures ('fingers'). After the crash the cycle repeats again starting from a state of low pressure/low current. Notice that, unlike in the conventional peeling-ballooning cycle, the starting point for the new cycle is not to be located at the kink stability boundary because the end of the crash is determined by processes related to the non-linear ballooning and not to the kink instability. According to this new peeling-ballooning cycle, the ELM precursors (kink or peeling-ballooning modes) observed at JET certainly play an important role in the cycle but are not directly involved in the ELM event itself.

The picture is not yet complete. Depending on the individual discharge conditions WB modes might be more or less effective in delaying the pressure build-up with respect to the peeling and ballooning stability limits. The three situations that can arise are depicted in figure 18. Curve 1 corresponds to the case of relatively strong WB modes, where the kink limit is reached well before the ELM. As there is a rather large gap to the ideal ballooning limit, the ELM precursors will be observed over longer times in that case. In the case of slightly less effective WB modes, the pedestal state might reach the stability limits in the vicinity of the upper right corner of the stability triangle (curve 2). The ELM precursors are then only shortlived, and the ELM crash occurs shortly after their appearance. This case is often observed at JET, where the shorter precursors last of the order of 0.5-2 ms, and the ELM occurs while the precursor amplitudes are still growing. An example has been shown in figures 1 and 11 , prior to the first ELM. Due to their shortness these precursors cannot affect significantly the evolution of the pedestal build-up through WB mode suppression. The hypothesis is that the radial displacements associated with the precursor oscillations themselves give rise to local transient steepenings of the pedestal. Since in the upper right corner of the stability triangle the pedestal is already close to the ideal ballooning limit, the oscillations may cause the pedestal to cross the ideal ballooning limit, where the ELM is triggered. Finally, the pedestal evolution given by the curve 3 in figure 18 corresponds to the case where the ideal ballooning limit is reached directly. No ELM precursors are then observed, and the ELM is directly triggered. 


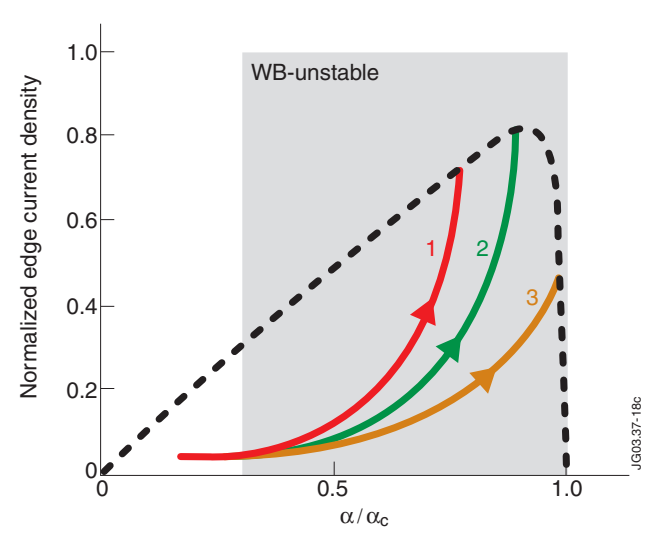

Figure 18. The three possible situations that may arise, depending on the WB amplitude and discharge conditions. Curve 1 corresponds to the case of relatively strong WB modes, where the kink limit is reached well before the ELM. ELM precursors will be observed over longer times in that case. In the case of slightly less effective WB modes, the pedestal state might reach the stability limits close to the upper right corner of the stability triangle (curve 2). The ELM occurs then shortly after the appearance of ELM precursors. The pedestal evolution given by the curve 3 corresponds to the case where the ideal ballooning limit is reached directly. No ELM precursors are then observed. The latter case may arise with rather weak WB modes, or in discharges where strong gas fuelling limits the amount of edge current.

There are at least two situations where this may occur. First, if the WB modes are rather weak, and second, if strong gas puffing is applied. As modelling calculations predict [29], the increase in edge collisionality associated with the gas puffing leads to a decrease in edge current density, keeping the pedestal state away from the kink limit. This is corroborated by experiment because ELM precursors are currently not observed at JET with strong gas puffing, even in discharges at high triangularity with strong WB modes and a low type-I ELM frequency (mixed type-I/type-II ELM regime).

\section{Summary and discussion}

Several features of an edge instability very commonly observed in JET and named the WB mode have been presented. WB modes are observed in JET exclusively in the H-mode regime and are thus apparently linked to the existence of a pedestal. They are composed of several bands of continuously bursting magnetic fluctuation, whose frequency evolution tends to follow the evolution of the edge electron temperature measured near the pedestal top. This is probably related to the strong diamagnetic drift associated with the large pedestal gradients. Evidence for their involvement in the pedestal and ELM dynamics has been presented. It has been shown that WB modes influence the build-up of the pedestal temperature (and, linked to this, also of the pedestal pressure) of the electrons, most likely through a continuous release of energy across the plasma boundary. This is further consistent with the observed correlation of WB mode activity with the time, $\Delta t_{\mathrm{ELM}}$, between consecutive ELMs. An exclusive interaction between the type-I ELM precursor modes and the WB modes has been identified. The onset of type-I ELM precursors is associated with a weakening, or even an inhibition, of the WB modes. The reduced WB mode amplitude often results in a faster build-up of the electron pressure at the pedestal, until it is finally interrupted by the ELM event. The enhancement of WB modes through gas puffing at high triangularity may provide an explanation for the occurrence of the new regime identified on JET that has been called the mixed type-I/type-II ELM regime. 
WB modes provide the enhanced turbulence identified in $[25,26]$ as a characteristic signature of the mixed type-I/type-II ELM regimes, but it needs to be emphasized that WB modes are also present in regimes regarded as pure type-I ELM regimes. A modified version of the peelingballooning cycle that includes the WB physics has been proposed. It predicts the type-I ELMs to be triggered at the ideal ballooning limit rather than the kink-/peeling-boundary and is therefore consistent with the experimental observation that the ELM precursors (thought to be kink modes/peeling-ballooning modes) seem not to trigger the ELM on JET. The precursors do however favour an earlier onset of the ELM through two effects: first, through the observed suppression of the WB modes. Second, the radial displacements associated with the precursor oscillations may cause transient local steepenings of the pedestal pressure gradient and thus favour the onset of ideal ballooning modes as a secondary instability.

Several open questions remain. Certainly, the most relevant one concerns the nature of the WB modes, which remains an unresolved issue. WB modes show no radial phase inversions, and hence magnetic islands can be excluded. The list of candidates can be further reduced by the identification of WB-driving or -stabilizing forces. Current-driven modes can be excluded from the observed WB mode enhancement through gas puffing (at high triangularity). For current-driven modes a weakening would have been expected due to the reduced edge current density associated with the high edge collisionality. The example discussed in section 3 , where WB modes become stabilized by the arrival of a large sawtooth heat pulse at the plasma edge, serves to exclude ideal pressure-driven modes. In that case, the sawtooth heat pulse causes a sudden increase in edge temperature, while the edge density remains nearly unaffected. Therefore, the WB suppression coincides with a sudden increase in the overall edge pressure, which is difficult to justify in terms of an ideal pressure-driven mode. Nevertheless, the increase in edge temperature implies a decrease in edge resistivity, and thus resistive pressure-driven modes cannot be excluded. Resistive ballooning modes (RBMs) have been regarded as a possible candidate to explain the WB modes in [7], where several arguments in favour of or against this are discussed in detail. In particular, RBMs have twisting parity [32] and certain branches are expected to propagate in the direction of the electron diamagnetic drift [33], like the WB modes, but on the other hand the $n$-numbers observed in the experiment are rather low. A ballooning character of the WB modes was inferred in [7] from the observed amplitude asymmetry measured by coils on the in-board and out-board sides of the plasma. However, recent modelling calculations performed with MISHKA [34] in real JET geometry have shown that mode-induced field perturbations are subject to higher damping on the HFS than on the LFS and that this may introduce a strong inboard-outboard asymmetry in the magnetically measured signal amplitudes even if the mode itself does not have ballooning character (more details are given in [8]). Therefore, the question whether WB modes have ballooning character or not is left open.

Kelvin-Helmholtz (KH) instabilities [35] may provide an alternative explanation for the WB modes. KH modes are driven by the shear of the parallel plasma velocity, which is expected to be large at the $\mathrm{H}$-mode barrier, while density and temperature gradients provide a stabilizing effect. They are not expected to be highly localized but to have rather broad radial extent, and are not bound to a certain rational surface; that is, their $m$ - and $n$-numbers do not have to correspond to the local value of the safety factor. However, the $m$-numbers that have been obtained for the WB bands with $n=-1$ do correspond to a rational surface localized close to the plasma boundary. The stabilization of WB modes by the ELM precursors could be easily explained in terms of KH because one expects the ELM precursors to affect the rotational shear at the edge. In similar terms, the arrival of a large sawtooth heat pulse at the plasma edge should be able as well to influence the parallel velocity shear and thus possibly stabilize the WB modes. Recent calculations that take into account the stabilizing effect of 
both the temperature and the density gradients on the $\mathrm{KH}$ modes show that they propagate (in the frame where $E_{r}=0$ ) in the direction of the electron diamagnetic drift, with a frequency that is a fraction of the electron diamagnetic frequency [36].

From the point of view of ELM and pedestal physics, it is desirable to ascertain whether MHD activity comparable with JET's WB modes is observed elsewhere. Taking into account how commonly WB modes are observed at JET, one might expect them to be present also in other machines. It is unlikely that WB modes are related to the quasi-coherent mode (QCM) observed during the EDA regime [37] of Alcator C-Mod, although there are some similarities. In particular, both modes occur at the plasma edge, rotate in the direction of the electron diamagnetic drift and seem to lead to a continuous loss of energy into the SOL [38]. There are, however, three major differences. First, the observed toroidal mode numbers of the QCM are in the range $n=15-18$ [39], which is much larger than those of the WB modes. Second, WB modes are currently composed of several bands of magnetic fluctuation activity, while for the QCM it is only a single band. Third, unlike the WB modes, the QCM is not observed in other $\mathrm{H}$-mode regimes, such as in standard ELM-free H-modes.

With the perspective on ITER operation and the avoidance of large transient heat loads onto the divertor tiles, the achievement of a stationary high confinement regime combined with small ELMs is certainly desirable. These conditions have been established in the type-II or 'grassy' ELM regimes of DIII-D [40], JT-60U [41] and ASDEX-U [27]. At JET, this could not be achieved yet, and it is questionable whether a mere further enhancement of the WB activity will be sufficient to obtain a pure type-II ELM regime. As shown in section 3, the WB modes are potentially capable of bringing the edge electron temperature to saturation. However, the edge density is not greatly affected by them but keeps increasing, at least in the gas puffed discharges. This highlights a serious problem because without a constant pedestal pressure a steady state regime will not become possible. It might be that WB modes on their own are not sufficient but that additional effects concerning the edge-related MHD have to come into play. A thorough comparison from the MHD point of view of the small ELM regimes obtained in other tokamaks is likely to clarify whether these rely on WB-like activity and whether there is a key ingredient that is missing in JET. This is intended for future work.

\section{Acknowledgments}

This work has been performed under the European Fusion Development Agreement. One author (CPP) wants to acknowledge the financial support of the Dutch Research Organisation NWO.

\section{References}

[1] Zohm H 1996 Plasma Phys. Control. Fusion 38105

[2] Connor J W 1998 Plasma Phys. Control. Fusion 40191

[3] Connor J W 1998 Plasma Phys. Control. Fusion 40531

[4] Leonard A W et al 1999 J. Nucl. Mater. 266-269 109

[5] Janeschitz G 2001 J. Nucl. Mater. 290-293 1

[6] Connor J W, Hastie R J and Wilson H R 1998 Phys. Plasmas 52687

[7] Smeulders P et al 1999 Plasma Phys. Control. Fusion 411303

[8] Perez C P et al Type-I ELM precursor modes in JET, EFDA-JET Preprint EFD-P(02)11 Nucl. Fusion submitted

[9] Nave M F F et al 1997 Nucl. Fusion 37809

[10] Huysmans G T A, Hender T and Alper B 1998 Nucl. Fusion 38179

[11] Merezhkin V G 1978 Sov. J. Plasma Phys. 4152

[12] Klueber O et al 1991 Nucl. Fusion 31907

[13] Buttery R J et al 2003 Nucl. Fusion 4369 
[14] JET Team (presented by Jones T T C) 1995 Plasma Phys. Control. Fusion 37 A359

[15] Costley A E 1991 Microwave reflectometry Diagnostics for Contemporary Fusion Experiments (International School of Plasma Physics, Varenna) ed P E Stott et al (Bologna: Editrice Compositori) p 113

[16] Sips A C C and Kramer G J 1993 Plasma Phys. Control. Fusion 35743

[17] Gowers C et al 1995 Rev. Sci. Instrum. 66471

[18] Hazeltine R D 1974 Phys. Fluids 17961

[19] Kim Y B, Diamond P H and Groebner R J 1991 Phys. Fluids B 32050

[20] Braithwaite G et al 1989 Rev. Sci. Instrum. 602825

[21] Saibene G et al 1999 Nucl. Fusion 391133

[22] Loarte A et al 2001 Proc. 28th EPS Conf. on Controlled Fusion and Plasma Physics (Madeira, 2001) P3.005

[23] Saibene G et al 2001 Proc. 28th EPS Conf. on Controlled Fusion and Plasma Physics (Madeira, 2001) P3.002

[24] Loarte A et al 2002 Plasma Phys. Control. Fusion 441815

[25] Saibene G et al 2002 Plasma Phys. Control. Fusion 441769

[26] Becoulet M et al 2002 Plasma Phys. Control. Fusion 44103

[27] Stober J et al 2001 Nucl. Fusion 411123

[28] Snyder P B et al 2002 Phys. Plasmas 92037

[29] Parail V et al 2002 Proc. 19th IAEA Fusion Energy Conf. (Lyon, 2002) IAEA-CN-94-TH/P3-08

[30] Hurricane O A, Fong B H and Cowley S C 1997 Phys. Plasmas 43565

[31] Fong B H 1999 Metastable and explosive properties of ballooning modes in laboratory and space plasmas PhD Thesis available at http://www.asp.ucar.edu/ bhlfong/thesis.pdf

[32] Strauss H R 1981 Phys. Fluids 242004

[33] Diamond P H et al 1985 Nucl. Fusion 281116

[34] Mikhailovskii A B et al 1997 Plasma Phys. Rep. 23844

[35] D’Angelo N 1965 Phys. Fluids 81748

[36] Rogister A L, Singh R and Kaw P K On ion temperature and parallel velocity shear instabilities Phys. Plasmas submitted

[37] Greenwald M et al 1997 Nucl. Fusion 37793

[38] Snipes J A et al 2001 Plasma Phys. Control. Fusion 43 L23

[39] Snipes J A et al 2002 Proc. 29th EPS Conf. on Controlled Fusion and Plasma Physics (Montreux, 2002) P1.057

[40] Ozeki T et al 1990 Nucl. Fusion 301425

[41] Kamada Y et al 2002 Plasma Phys. Control. Fusion 44 A279 